\title{
Weak normality of families of meromorphic mappings and bubbling in higher dimensions
}

\author{
SERGEy IVAShKovich AND FETHi NeJI
}

\begin{abstract}
Our primary goal in this paper is to understand whether the sets of normality of families of meromorphic mappings between general complex manifolds are pseudoconvex or not. It turns out that the answer crucially depends on the type of convergence one is interested in. We examine three natural types of convergence introduced by one of us earlier and prove pseudoconvexity of sets of normality for a large class of target manifolds for the so called weak and gamma convergencies. Furthermore we determine the structure of the exceptional components of the limit of a weakly/gamma but not strongly converging sequence, they turn to be rationally connected. This observation allows to determine effectively when a weakly/gamma converging sequence fails to converge strongly. An application to the Fatou sets of meromorphic self-maps of compact complex surfaces is given.
\end{abstract}

Mathematics Subject Classification (2010): 32H04 (primary); 32H50, 32Q45 (secondary).

\section{Introduction}

\subsection{Convergence of meromorphic mappings}

When one works with sequences of meromorphic functions and, more generally, mappings one finds himself bounded to consider several notions of their convergence. Some of these notions were introduced in [10] and [18], we shall recall the essentials below. An important question is: what can be said about the maximal open sets where the given sequence converges? It occurs that pseudoconvexity or not of domains of convergence/normality in the case of meromorphic mappings crucially depends on the type of convergence one is looking for.

Now let us briefly describe the ways one can define what does it mean that a sequence $\left\{f_{k}\right\}$ of meromorphic mappings between complex manifolds $U$ and $X$ converges. We start with the most obvious one. A sequence $\left\{f_{k}\right\}$ of meromorphic mappings between complex manifolds $U$ and $X$ is said to converge strongly to a meromorphic map $f$ if the graphs $\Gamma_{f_{k}}$ converge over compacts in $U$ to the graph 
$\Gamma_{f}$ in Hausdorff metric. Our first result shows that $\Gamma_{f_{k}}$ then converge to $\Gamma_{f}$ in a stronger topology of cycles.

Theorem 1.1. If $f_{k}$ strongly converge to $f$ then for every compact $K \Subset U$ the volumes $\Gamma_{f_{k}} \cap(K \times X)$ are uniformly bounded and therefore $\Gamma_{f_{k}}$ converge to $\Gamma_{f}$ in the topology of cycles.

This type of convergence is natural and has some nice features. For example the strong limit of a sequence of holomorphic maps is holomorphic and vice versa, if the limit $f$ is holomorphic then for every compact $K \Subset U$ all $f_{k}$ for $k \gg 1$ are holomorphic in a neighborhood of $K$ and uniformly converge there to $f$. This statement was called the Rouché Principle in [18].

But strong convergence has also some disadvantages. The first, crucial for us is the fact that the sets of strong convergence, i.e., maximal open subsets of $U$ where a given sequence converges strongly on compacts, are not pseudoconvex in general. Moreover, the sets of strong normality (see later on) of families of meromorphic mappings can be just arbitrary, see Example 2.8. Also if one takes $X=\mathbb{P}^{N}$ the "most immediate" notion of convergence does not correspond to the strong one.

Therefore in [18] along with the notion of strong convergence we proposed two weaker ones. We say that $f_{k}$ converge weakly to $f$ if they converge strongly to $f$ on compacts outside of some analytic set $A$ in $U$ of codimension at least two. It turns out that this $A$ can be taken to be the set $I_{f}$ of points of indeterminacy of the limit map $f$ and then for every compact $K$ in $U \backslash I_{f}$ all weakly converging to $f$ mappings $f_{k}$ will be holomorphic on $K$ (for $k$ big enough) and converge to $f$ uniformly on $K$, see Remark 3.2.

One more notion of convergence from [18], which we need to recall here, is the gamma convergence ( $\Gamma$-convergence). We say that $f_{k}$ gamma-converge to $f$ if they strongly converge to $f$ outside of an analytic set (now it can be of codimension one) and for every divisor $H$ in $X$ and every compact $K \Subset U$ the intersections $f_{k}^{*} H \cap K$ have bounded volume counted with multiplicities, see more about the last condition in section 3.2 .

Remark 1.2. Strong convergence (or $s$ - convergence) will be denoted by $f_{k} \rightarrow f$, the weak one (or $w$ - convergence) as $f_{k} \rightarrow f$, and $\Gamma$ - convergence as $f_{k} \stackrel{\Gamma}{\rightarrow} f$. Note that in the second and third definitions we suppose that the limit $f$ is defined and meromorphic on the whole of $U$ if, even, the convergence takes place only on some part of $U$. In the first case the limit exists on the whole of $U$ automatically.

For the better understanding of these notions let us give a description of the listed types of convergence in the case when $X$ is projective, i.e., imbeds into $\mathbb{P}^{N}$ for some $N$. In that special case the notions of convergence listed above permit an explicit analytic description as follows. Every meromorphic mapping $f$ with values in $\mathbb{P}^{N}$ can be locally represented by an $(N+1)$-tuple of holomorphic functions

$$
f(z)=\left[f^{0}(z): \ldots: f^{N}(z)\right]
$$


where not all of $f^{0}, \ldots, f^{N}$ are identically zero, see section 4 . More precisely, if $f: U \rightarrow \mathbb{P}^{N}$ is a meromorphic mapping then for every point $x_{0} \in U$ there exists a neighborhood $V \ni x_{0}$ and holomorphic functions $f^{0}, \ldots, f^{N}$ in $V$ satisfying (1.1). If the zero sets of $f^{j}$ contain a common divisor then we can divide all $f^{j}$ by its equation and get a representation such that $\operatorname{GCD}\left(f^{1}, \ldots, f^{N}\right)=1$ in every $\mathcal{O}_{x}$, $x \in V$. In that case the indeterminacy set of $f$ is

$$
I_{f} \cap V=\left\{z \in V: f^{0}(z)=\ldots=f^{N}(z)=0\right\}
$$

and has codimension at least two. Representation (1.1) satisfying (1.2) is called reduced. We shall prove the following:

Theorem 1.3. Let $\left\{f_{k}\right\}$ be a sequence of meromorphic mappings from a complex manifold $U$ to $\mathbb{P}^{N}$. Then:

i) $f_{k} \stackrel{\Gamma}{\rightarrow} f$ if and only if for any point $x_{0} \in U$ there exist a neighborhood $V \ni x_{0}$, reduced representations $f_{k}=\left[f_{k}^{0}: \ldots: f_{k}^{N}\right]$ and not necessarily reduced representation $f=\left[f^{0}: \ldots: f^{N}\right]$ such that for every $0 \leq j \leq N$ the sequence $f_{k}^{j}$ converges to $f^{j}$ uniformly on $V$;

ii) $f_{k} \rightarrow f$ if and only if $f_{k} \stackrel{\Gamma}{\rightarrow} f$ and the limit representation $f=\left[f^{0}: \ldots: f^{N}\right]$ is reduced;

iii) $f_{k} \rightarrow f$ if and only if $f_{k} \rightarrow f$ and corresponding non-pluripolar MongeAmpère masses converge, i.e., for every $1 \leq p \leq n=\operatorname{dim} U$ one has

$$
\left(d d^{c}\|z\|^{2}\right)^{n-p} \wedge\left(d d^{c} \ln \left\|f_{k}\right\|^{2}\right)^{p} \rightarrow\left(d d^{c}\|z\|^{2}\right)^{n-p} \wedge\left(d d^{c} \ln \|f\|^{2}\right)^{p}
$$

weakly on compacts in $U$.

Here in (1.3) we suppose that $V=\Delta^{n}, z_{1}, \ldots, z_{n}$ are standard coordinates and $\|f\|^{2}=\left|f^{0}\right|^{2}+\ldots+\left|f^{N}\right|^{2}$, i.e., $d d^{c} \ln \|f\|^{2}$ is the pullback of the Fubini-Study form by $f$. Non-pluripolar MA mass of $\ln \|f\|^{2}$ of order $p$ in $V$ here means

$$
\int_{V \backslash I_{f}}\left(d d^{c}\|z\|^{2}\right)^{n-p} \wedge\left(d d^{c} \ln \|f\|^{2}\right)^{p},
$$

where $I_{f}$ is given by (1.2), i.e., is the indeterminacy set of $f$.

Remark 1.4. a) Reducibility or not of the limit representation $f=\left[f^{0}: \ldots: f^{N}\right]$ in this theorem does not depend on the choice of converging representations $f_{k}=$ $\left[f_{k}^{0}: \ldots: f_{k}^{N}\right]$, provided they are taken to be reduced (the last can be assumed always). Indeed, any other reduced representation of $f_{k}$ has the form $f_{k}=\left[g_{k} f_{k}^{0}\right.$ : $\ldots: g_{k} f_{k}^{N}$, where $g_{k}$ are holomorphic and nowhere zero. If the newly chosen representations converge to some representation of $f$ then $g_{k}$ must converge, say to $g$, and this $g$ is nowhere zero by Rouché's theorem. Therefore the obtained 
representation of the limit is $f=\left[g f^{0}: \ldots: g f^{N}\right]$ and it is reduced if and only if $f=\left[f^{0}: \ldots: f^{N}\right]$ was reduced.

b) The case when the representation $f=\left[f^{0}: \ldots: f^{N}\right]$ of the limit is not necessarily reduced was studied for mappings with values in $\mathrm{P}^{N}$ by $\mathrm{H}$. Fujimoto in [10], who called it meromorphic, or $m$-convergence. According to the part (i) of our theorem it turns out that our $\Gamma$-convergence (in the case of $X=\mathbb{P}^{N}$ ) is equivalent to $m$-convergence of Fujimoto.

\subsection{Sets of normality and Bloch-Montel type criterion}

In this paper we consider two classes of complex manifolds: projective and Gauduchon, the last is the class of complex manifolds carrying a $d d^{c}$-closed metric form a Gauduchon form. Let $\mathcal{F}$ be a family of meromorphic mappings between complex manifolds $U$ and $X . \mathcal{F}$ is said to be strongly/weakly or gamma normal if from every sequence of elements of $\mathcal{F}$ one can extract a subsequence converging on compacts in $U$ in the corresponding sense. The maximal open subset $\mathcal{N}_{\mathcal{F}} \subset U$ on which $\mathcal{F}$ is normal is called the set of normality. As it was already told the sets of strong normality could be arbitrary. In Subsection 3.1 we prove the following:

Theorem 1.5. Let $U$ be a domain in a Stein manifold $\hat{U}$ such that $\hat{U}$ is an envelope of holomorphy of $U$ and let $f_{k}: \hat{U} \rightarrow X$ be a weakly converging on $U$ sequence of meromorphic mappings with values in a disk-convex complex manifold $X$. Then:

(a) If the weak limit $f$ on $f_{k}$ meromorphically extends from $U$ to $\hat{U}$ then $f_{k}$ weakly converge to $f$ on the whole of $\hat{U}$.

(b) If, in addition, the manifold $X$ carries a pluriclosed metric form then the weak limit $f$ of $f_{k}$ meromorphically extends to $\hat{U}$ and then the part (a) applies.

As a result the sets of weak normality are locally pseudoconvex provided the target is disk-convex and Gauduchon. Recall that an open subset $\mathcal{N}$ of a complex manifold $U$ is called locally pseudoconvex if for every point $p \in \partial \mathcal{N}$ there exists a Stein neighborhood $V$ of $p$ in $U$ such that $V \cap \mathcal{N}$ is Stein.

Corollary 1.6. Let $\mathcal{F} \subset \mathcal{M}(U, X)$ be a family of meromorphic mappings from a complex manifold $U$ to a disk-convex Gauduchon manifold $X$. Then the set of weak normality $\mathcal{N}_{\mathcal{F}}$ of $\mathcal{F}$ is locally pseudoconvex. If $\mathcal{F}=\left\{f_{k}\right\}$ is a sequence then the set of its weak convergence is locally pseudoconvex.

Remark 1.7. This corollary clearly follows from Theorem 1.5 . Sets of $\Gamma$-normality are also locally pseudoconvex under the same assumptions, see Proposition 3.10 in Section 2.

As one more supporting argument in favor of weak convergence we prove in Section 5 the following normality criterion. 
Theorem 1.8. Let $\left\{H_{i}\right\}_{i=0}^{d}, d \geq 1$, be hypersurfaces in projective manifold $X$ such that $Y:=X \backslash \bigcup_{i=0}^{d} H_{i}$ is hyperbolically imbedded to $X$. Let $\mathcal{F}$ be a family of meromorphic mappings from a complex manifold $U$ to $X$ such that:

i) for every $i=0, \ldots, d$ and every compact $K \Subset U$ the volumes $f^{*} H_{i} \cap K$ counted with multiplicities are uniformly bounded for $f \in \mathcal{F}$;

ii) $\mathcal{F}$ uniformly separates every pair $H_{i}, H_{j}, 0 \leq i<j \leq d$.

Then the family $\mathcal{F}$ is weakly normal on $U$.

Conditions i) and ii) are explained in Section 5, they are intuitively clear and more or less necessary. The classically known case of a system of divisors with hyperbolically imbedded complement is $2 N+1$ hypersurfaces in $\mathbb{P}^{N}$ in general position - Theorem of Bloch, see [13]. A criterion for $m$-normality (i.e., $\Gamma$-normality in our sense) was given by Fujimoto in [10].

\subsection{Rational connectivity of the exceptional components of the limit}

Strong convergence obviously implies the weak one and the latter implies the gamma-convergence, see Remark 3.6:

$$
\text { s-convergence } \Longrightarrow \text { w-convergence } \Longrightarrow \Gamma \text { - convergence. }
$$

Our second principal task in this paper is to understand what obstructs a weakly/ gamma converging sequence to converge strongly. The problem is that by Theorem 1.1 the volumes of graphs of a strongly converging sequence are uniformly bounded over compacts in the source. When dimension $n$ of the source $U$ is two and $X$ is Kähler the volumes of the graphs of a weakly converging sequence are still bounded, see [18]. The same is true if is $X$ an arbitrary compact complex surface (and again $\operatorname{dim} U=2$ ), see [32]. We shall say more about this in Section 6. But this turns out not to be the case starting from dimension three, i.e., the volumes of graphs of a weakly converging sequence can diverge to infinity over compacts of $U$. Via (1.3) this turns out to be a geometric counterpart of a well known discontinuity of Monge-Ampère masses, see Example 6.1 in Section 4. Nevertheless for a sequence $\Gamma_{f_{k}}$ of $\Gamma$-converging meromorphic graphs we can consider the Hausdorff limit $\hat{\Gamma}$ (its always exists after taking a subsequence). Set $\Gamma:=\overline{\hat{\Gamma} \backslash \Gamma_{f}}$, where $\Gamma_{f}$ is the graph of the limit map $f$, and call $\Gamma$ a bubble. For $a \in \gamma:=\operatorname{pr}_{1}(\Gamma)$ set $\Gamma_{a}:=\operatorname{pr}_{2}\left(\operatorname{pr}_{1}^{-1}(a) \cap \Gamma\right)$, here $\mathrm{pr}_{1}$ and $\mathrm{pr}_{2}$ are natural projections, see Section 2 . We prove the following statement.

Theorem 1.9. Let $X$ be a disk-convex Gauduchon manifold and let $f_{k}: U \rightarrow X$ be a weakly converging sequence of meromorphic mappings which does not converge strongly. Then for every point $a \in \gamma$ the fiber $\Gamma_{a}$ is rationally connected. If $X$ is, moreover, projective then the same is true also for $\Gamma$-converging sequences. 
Here by saying that a closed subset $\Gamma_{a}$ of a complex manifold is rationally connected we mean that every two distinct points $p, q \in \Gamma_{a}$ can be connected by a chain of rational curves which is entirely contained in $\Gamma_{a}$, see Section 7 for more details.

\subsection{Fatou sets of meromorphic self-maps}

Families of a special interest are the families of iterates $f^{n}:=f \circ \ldots \circ f$ of some fixed meromorphic self-map of a compact complex manifold $X$. The maximal open subset $X$ where $\left\{f^{n}\right\}$ is relatively compact is called the Fatou set of $f$. Depending on the sense of convergence that one wishes to consider one gets different Fatou sets: strong, weak or gamma Fatou sets. We denote them as $\Phi_{s}, \Phi_{w}$ and $\Phi_{\Gamma}$ respectively, their dependance on $f$ will be clear from the context.

Corollary 1.10. Let $f$ be a meromorphic self-map of a compact complex surface. Then the weak Fatou set $\Phi_{w}$ of $f$ is locally pseudoconvex. If $\Phi_{s}$ is different from $\Phi_{w}$ then:

a) $X$ is bimeromorphic to $\mathbb{P}^{2}$;

b) $\Phi_{w}=X \backslash C$, where $C$ is a rational curve in $X$;

c) the weak limit of any weakly converging subsequence $\left\{f^{n_{k}}\right\}$ of iterates is a degenerate map of $X$ onto $C$.

It should be pointed out that our Fatou sets are different from the Fatou sets as they were considered in [8]. In [8] the Fatou set of $f$ is the maximal open subset $\Phi$ of $X \backslash \overline{\bigcup_{n=0}^{\infty} f^{-n}\left(I_{f}\right)}$ where the family $\left\{f^{n}\right\}$ is equicontinuous (remark that on $X \backslash \overline{\bigcup_{n=0}^{\infty} f^{-n}\left(I_{f}\right)}$ all iterates are holomorphic). If, for example, $f: \mathbb{P}^{2} \rightarrow \mathbb{P}^{2}$ is the Cremona transformation $\left[z_{0}: z_{1}: z_{2}\right] \rightarrow\left[z_{1} z_{2}: z_{0} z_{2}: z_{0} z_{1}\right]$ then $\Phi_{s}=\Phi_{w}=$ $\Phi_{\Gamma}=\mathbb{P}^{2}$ but $\Phi=\mathbb{P}^{2} \backslash\{$ three lines $\}$. In Subsection 8.2 an example of higher degree and with an interesting dynamics on the indeterminacy set is given. This is one more instance which shows how crucially can change a picture when the notion of convergence changes.

Notes

1. Let us make a final note about the goals of this paper. On our opinion the most interesting information about a converging sequence of meromorphic mappings is concentrated near the "limit" of their indeterminacy sets. We describe the most reasonable (in our opinion) notions of convergence of meromorphic mappings and conclude that the weak one is the most appropriate. At the same time we detect that if a weakly/gamma converging sequence does not converge strongly then this imposes very serious restrictions on the target manifold (it is forced to contain many rational curves). In some cases (ex. iterations) this puts strong constraints also on the sequence itself.

2. Domains of convergence of holomorphic functions of several variables were, probably, for the first time considered by G. Julia in [24]. In [24] and then in [6] it was proved that these domains are (in some sense) pseudoconvex. Domains of 
convergence of meromorphic functions of several variables were studied in [38] and then in [37]. In these early papers convergence was understood as holomorphic (i.e., uniform) convergence outside of the union of indeterminacy sets of meromorphic mappings in question.

ACKNOWLEDGEMENTS. We are grateful to Alexander Rashkovskii for explaining to us the Example 6.1 with unbounded Monge-Ampère masses.

\section{Topologies on the space of meromorphic mappings}

\subsection{Complex manifolds and meromorphic mappings}

Our manifolds will be Hausdorff and countable at infinity if the opposite is not explicitly stated. We shall also everywhere suppose that they are disk-convex.

Definition 2.1. A complex manifold $X$ is called disk-convex if for every compact $K \Subset X$ there exists a compact $\hat{K}$ such that for every $h \in \mathcal{O}(\Delta, X) \cap \mathcal{C}(\bar{\Delta}, X)$ such that $h(\partial \Delta) \subset K$ one has $h(\bar{\Delta}) \subset \hat{K}$.

The minimal such $\hat{K}$ is called the disk envelope of $K$. Let $X$ be equipped with some Hermitian metric $h$. By $\omega_{h}$ denote the $(1,1)$-form canonically associated with $h$. We say that the metric $h$ is $d$-closed or Kähler if $d \omega_{h}=0$. We say that $h$ is pluriclosed or Gauduchon if $d d^{c} \omega_{h}=0$. In [11] it was proved that on a compact complex surface every Hermitian metric is conformally equivalent to the unique $d d^{c}$-closed one.

Remark 2.2. We shall need only the existence of such metric forms on compact complex surfaces and this can be proved by duality: non existence of a positive $d d^{c}$ closed $(1,1)$-form is equivalent to the existence of a non-constant plurisubharmonic function. The latter on a compact complex manifold is impossible.

We also fix some metric form $\omega_{1}$ on $U$. In the case of a polydisk $U=\Delta^{n}$ we will work with the standard Euclidean metric $e$. The associated form will be denoted by $\omega_{e}=d d^{c}\|z\|^{2}=\frac{i}{2} \sum_{j=1}^{n} d z_{j} \wedge d \bar{z}_{j}$. By $\operatorname{pr}_{1}: U \times X \longrightarrow U$ and $\operatorname{pr}_{2}: U \times X \longrightarrow X$ denote the projections onto the first and second factors. On the product $U \times X$ we consider the metric form $\omega=\operatorname{pr}_{1}^{*} \omega_{1}+\operatorname{pr}_{2}^{*} \omega_{h}$.

A meromorphic mapping $f$ between complex manifolds $U$ and $X$ is defined by an irreducible analytic subset $\Gamma_{f} \subset U \times X$ such that

- the restriction $\left.\mathrm{pr}_{1}\right|_{\Gamma_{f}}: \Gamma_{f} \rightarrow U$ of the natural projection to $\Gamma_{f}$ is a proper modification, i.e., is proper and generically one to one.

$\Gamma_{f}$ is called the graph of $f$. Due to the irreducibility of $\Gamma_{f}$ and the Remmert proper mapping theorem the set of points over which $\mathrm{pr}_{1}$ is not one to one is an analytic subset of $U$ of codimension at least two. This set is called the set of points of indeterminacy of $f$ and is usually denoted as $I_{f}$. Therefore an another way to define 
a meromorphic mapping $f$ between complex manifolds $U$ and $X$ is by considering a holomorphic map $f: U \backslash A \rightarrow X$, where $A$ is an analytic subset of $U$ of codimension at least two, such that the closure $\Gamma_{f}$ of its graph is an analytic subset of the product $U \times X$ satisfying the condition above. Remark that the analyticity of the closure of the holomorphic graph is not automatic. Think about the natural projection $f: \mathbb{C}^{2} \backslash\{0\} \rightarrow \mathbb{C}^{2} \backslash\{0\} / z \sim 2 z$ of $\mathbb{C}^{2} \backslash\{0\}$ onto a Hopf surface. The properness of the restriction of the projection $\mathrm{pr}_{1}$ to the closure is, unless $X$ is disk convex, not automatic too.

The volume of the graph $\Gamma_{f}$ of a meromorphic mapping $f$ is given by

$$
n ! \operatorname{Vol}\left(\Gamma_{f}\right)=\int_{\Gamma_{f}} \omega^{n}=\int_{\Gamma_{f}}\left(\operatorname{pr}_{1}^{*} \omega_{1}+\operatorname{pr}_{2}^{*} \omega_{h}\right)^{n}=\int_{U}\left(\omega_{1}+f^{*} \omega_{h}\right)^{n}
$$

where $n=\operatorname{dim} U$.

Remark 2.3. Let us make a few remarks concerning the notion of a meromorphic mapping.

a) If $V$ is a subvariety of $U$ such that $V \not \subset I_{f}$ then the restriction $\left.f\right|_{V}$ of $f$ to $V$ is defined by taking as its graph $\Gamma_{\left.f\right|_{V}}$ the irreducible component of the intersection $\Gamma_{f} \cap(V \times X)$ which projects onto $V$ generically one to one. Therefore $\Gamma_{\left.f\right|_{V}} \subset \Gamma_{f} \cap(V \times X)$ and the inclusion here is proper in general. The full image of a set $L \subset U$ under $f$ is defined as $f[L]:=\operatorname{pr}_{2}\left(\Gamma_{f} \cap[L \times X]\right)$.

b) It is probably worth to notice that $x \in I_{f}$ if and only if $\operatorname{dim} f[x] \geq 1$. This follows from the obvious observation that

$$
I_{f}=\operatorname{pr}_{1}\left(\left\{\left(x_{1}, x_{2}\right) \in \Gamma_{f}:\left.\operatorname{dim}_{\left(x_{1}, x_{2}\right)} \operatorname{pr}_{1}\right|_{\Gamma_{f}} ^{-1}\left(x_{1}\right) \geq 1\right\}\right) .
$$

c) If $\operatorname{dim} V=1$ then the irreducible component of $\Gamma_{f} \cap(V \times X)$ which projects onto $V$ is a curve. Since the projection is generically one to one it is on to one everywhere and therefore the restriction $\left.f\right|_{V}$ is necessarily holomorphic.

d) Let us give the sense to $f^{*} \omega_{h}$ in the formula (2.1). The first integral there has perfectly sense since we are integrating a smooth form over a complex variety. Denote by $I_{f}^{\varepsilon}$ the $\varepsilon$-neighborhood of the indeterminacy set $I_{f}$ of $f$. Then (2.1) shows that the limit

$$
\lim _{\varepsilon \rightarrow 0} \int_{U \backslash \bar{I}_{f}^{\varepsilon}}\left(\omega_{1}+f^{*} \omega_{h}\right)^{n}=\lim _{\varepsilon \rightarrow 0} \int_{U \backslash \bar{I}_{f}^{\varepsilon}} \sum_{p=0}^{n} C_{n}^{p} \omega_{1}^{n-p} \wedge f^{*} \omega_{h}^{p}
$$

exists. Therefore all $f^{*} \omega_{h}^{p}$ are well defined on $U$ as positive currents.

\subsection{Analytic cycles and currents}

Before turning to the notions of convergence of meromorphic mappings let us recall the natural topologies on the space of analytic subsets of a complex manifold. 
Recall that an analytic cycle of dimension $r$ in a complex manifold $Y$ is a formal sum $Z=\sum_{j} n_{j} Z_{j}$, where $\left\{Z_{j}\right\}$ is a locally finite sequence of reduced analytic subsets of pure dimension $r$ and $n_{j}$ are positive integers called multiplicities of $Z_{j}$. The set $|Z|:=\bigcup_{j} Z_{j}$ is called the support of $Z$. In our applications $Y$ will be $U \times X$ and $r$ will be the dimension $n=\operatorname{dim} U$. By a coordinate chart adapted to $Z$ we shall understand a relatively compact open set $V$ in $Y$ such that $V \cap|Z| \neq \varnothing$ together with a biholomorphism $j$ of $V$ onto a neighborhood $\tilde{V}$ of $\bar{\Delta}^{r} \times \bar{\Delta}^{q}$ in $\mathbb{C}^{r+q}, r+q=\operatorname{dim} Y$, such that $j^{-1}\left(\bar{\Delta}^{r} \times \partial \Delta^{q}\right) \cap|Z|=\emptyset$. We shall denote such chart by $(V, j)$. The image $j(Z \cap V)$ of the cycle $Z \cap V$ under biholomorphism $j$ is the image of the underlying analytic set together with multiplicities. Following Barlet and Fujiki, see [2] and [9], we call the quadruple $E=\left(V, j, \Delta^{r}, \Delta^{q}\right)$ a scale adapted to $Z$.

If pr: $\mathbb{C}^{r} \times \mathbb{C}^{q} \rightarrow \mathbb{C}^{r}$ is the natural projection, then the restriction pr $\left.\right|_{j(Z \cap V)}$ : $j(Z \cap V) \rightarrow \Delta^{r}$ is a branched covering of degree say $d$. This branched covering defines in a natural way a holomorphic mapping $\phi_{j, Z}: \Delta^{r} \rightarrow \operatorname{Sym}^{d}\left(\Delta^{q}\right)$ to the $d$-th symmetric power of $\Delta^{q}$ by setting $\phi_{j, Z}\left(z^{\prime}\right)=\left\{(\operatorname{pr} \mid j(Z \cap V))^{-1}\left(z^{\prime}\right)\right\}$. The latter denotes the unordered set of all preimages of $z^{\prime}$ under the projection in question. This construction, due to Barlet, allows to represent a cycle $Z \subset Y$ by a set of holomorphic maps $\phi_{j_{\alpha}, Z}: \Delta^{r} \rightarrow \operatorname{Sym}^{d}\left(\Delta^{q}\right)$, where $\left\{\left(V_{\alpha}, j_{\alpha}\right)\right\}$ is some open covering of $|Z|$ by adapted coordinate charts.

Definition 2.4. One says that $Z_{k}$ converges to $Z$ in the topology of cycles if for every coordinate chart $(V, j)$ adapted to $Z$ there exists $k_{0}$ such that $\forall k \geq k_{0}$ this chart will be adapted to $Z_{k}$ and the sequence of corresponding holomorphic mappings $\phi_{j, Z_{k}}$ converge to $\phi_{j, Z}$ uniformly on $\Delta^{r}$.

This defines a metrizable topology on the space $\mathcal{C}_{r}(Y)$ of $r$-cycles in $Y$. This topology is equivalent to the topology of currents: $Z_{k} \rightarrow Z$ if for any continuous $(r, r)$-form $\chi$ with compact support one has

$$
\int_{Z_{k}} \chi \rightarrow \int_{Z} \chi
$$

see [9]. It is also equivalent to the Hausdorff topology under an additional condition of boundedness of volumes. Recall that the Hausdorff distance between two subsets $A$ and $B$ of a metric space $(Y, \rho)$ is a number $\rho(A, B)=\inf \left\{\varepsilon: A^{\varepsilon} \supset B, B^{\varepsilon} \supset A\right\}$. Here by $A^{\varepsilon}$ we denote the $\varepsilon$-neighborhood of the set $A$, i.e. $A^{\varepsilon}=\{y \in Y$ : $\rho(y, A)<\varepsilon\}$.

Now, $Z_{k} \rightarrow Z$ if and only if for every compact $K \Subset Y$ there exists $C_{K}>0$ such that $\operatorname{Vol}_{2 r}\left(Z_{k} \cap K\right) \leq C_{K}$ and $Z_{k} \cap K \rightarrow Z \cap K$ with respect to the Hausdorff distance. This statement is the content of the Harvey-Shiffman's generalization of Bishop's compactness theorem. For the proof see [15]. We denote the space of $r$-cycles on $Y$ endowed with the topology described as above by $\mathcal{C}_{r}^{\text {loc }}(Y)$. 


\subsection{Strong convergence of meromorphic mappings}

Let $\left\{f_{k}\right\}$ be a sequence of meromorphic mappings of a complex manifold $U$ to a complex manifold $X$.

Definition 2.5. We say that $f_{k}$ converge strongly to a meromorphic map $f: U \rightarrow$ $X$ (s-converge) if the sequence of graphs $\Gamma_{f_{k}}$ converge over compacts to $\Gamma_{f}$ in Hausdorff metric, i.e., for every compact $K \Subset U$ one has $\Gamma_{f_{k}} \cap(K \times X) \stackrel{H}{\rightarrow}$ $\Gamma_{f} \cap(K \times X)$.

Now let us prove Theorem 1.1 from the Introduction, i.e., that Hausdorff convergence in the case of graphs implies the boundedness of volumes (over compacts) and therefore the convergence in the topology of cycles. Let us underline at this point that in this theorem one does not need to suppose anything on the target manifold $X$.

Proof of Theorem 1.1. The reason why Hausdorff convergence of graphs implies their stronger convergence in the topology of cycles is that, being the graphs, the analytic cycles $\Gamma_{f_{k}}$ converge to $\Gamma_{f}$ with multiplicity one. Now let us give the details. Let $a \in U \backslash I_{f}$ be a regular point of $f$ and set $b=f(a)$. Then we can find neighborhoods $D_{1} \ni a$ and $D_{2} \ni b$ biholomorphic to $\Delta^{n}, n=\operatorname{dim} U$ and $\Delta^{p}, p=$ $\operatorname{dim} X$ respectively such that $\Gamma_{f} \cap\left(\bar{D}_{1} \times \partial D_{2}\right)=\emptyset$. In particular $V=D_{1} \times D_{2}$ is an adapted chart for $\Gamma_{f}$, let $\left(V, j, \Delta^{n}, \Delta^{p}\right)$ be a corresponding scale. Here $j: V \rightarrow$ $\Delta^{n} \times \Delta^{p}$ is some biholomorphism. From Hausdorff convergence of $\Gamma_{f_{k}}$ to $\Gamma_{f}$ we see that for $k \gg 1 \Gamma_{f_{k}} \cap\left(\bar{D}_{1} \times \partial D_{2}\right)=\emptyset$. Therefore $\Gamma_{f_{k}} \cap\left(D_{1} \times D_{2}\right) \rightarrow D_{1}$ is a ramified covering (i.e., is proper) of some degree $d_{k}$. But $\Gamma_{f_{k}}$ is one to one over a generic point of $D_{1}$. Therefore $d_{k}=1$ and $\Gamma_{f_{k}} \cap V$ converge to $\Gamma_{f} \cap V$ as graphs (in particular as cycles). We proved that $f_{k}$ converge to $f$ on compacts of $U \backslash I_{f}$ as holomorphic mappings.

Let now $a \in I_{f}$ and take some $b \in f[a]$. As above take a neighborhood $V=D_{1} \times D_{2} \cong \Delta^{n} \times \Delta^{p}$ of $(a, b)$, where $a=0$ and $b=0$ in these coordinates. Denote by $\left(w^{\prime}, w^{\prime \prime}\right)$ the coordinates in $\Delta^{n} \times \Delta^{p}$. Perturbing the slope of coordinate $w^{\prime \prime}$ we can suppose that $\left(\{0\} \times \Delta^{p}\right) \cap \Gamma_{f}$ has 0 as its isolated point.

Remark 2.6. After perturbation of the slope of $w^{\prime \prime}$ the decomposition $j(V)=$ $\Delta^{n} \times \Delta^{p}$ will not correspond to the decomposition $U \times X$.

For sufficiently small $\varepsilon>0$ we take polydisks $\Delta_{\varepsilon}^{n}$ and $\Delta_{\varepsilon}^{p}$ in (perturbed) coordinates (actually only $w^{\prime \prime}$ needs to be perturbed). We get an adapted chart for $\Gamma_{f}$ which possed the following property:

i) $\tilde{V}:=j(V)$ writes as $\tilde{V}=\Delta^{n} \times \Delta^{p}, p=\operatorname{dim} X$.

ii) Local coordinates $\left(w^{\prime}, w^{\prime \prime}\right)$ of $\Delta^{n} \times \Delta^{p}$ enjoy the property that $z^{\prime}:=w^{\prime} \in \Delta^{n}$ is a local oordinate in $U$ (but $w^{\prime \prime}$ is not a local coordinate on $X$ ).

iii) $j\left(\Gamma_{f} \cap V\right) \rightarrow \Delta^{n}$ is a ramified covering of degree $d \geq 1$. 
Again from Hausdorff convergence of $\Gamma_{f_{k}}$ to $\Gamma_{f}$ we get that for all $k \gg 1$ the intersection $j\left(\Gamma_{f_{k}} \cap V\right)$ is a ramified covering of $\Delta^{n}$ of degree $d_{k}$. Obviously $d_{k} \geq d$ for $k \gg 1$. If for some subsequence $d_{k}>d$ we shall get a contradiction as follows. In that case some irreducible component of $\Gamma_{f} \cap V$ will be approached by $\Gamma_{f_{k}} \cap V$ at least doubly. Let $\Gamma$ stands for this irreducible component. Since $\operatorname{dim}\left[\Gamma_{f} \cap\left(I_{f} \times X\right)\right] \leq n-1$ (by irreducibility of $\Gamma_{f}$ ) we see that $\Gamma_{f_{k}}$ multiply approach every compact of $\Gamma \backslash\left(I_{f} \times X\right)$. Take a point $c \in \Gamma \backslash\left(I_{f} \times X\right)$ having a relatively compact neighborhood $W \subset \Gamma \backslash\left(I_{f} \times X\right)$ such that $\left.\operatorname{pr}_{1}\right|_{W}: W \rightarrow W_{0}$ is biholomorphic, i.e., $W$ is the graph of $f$ over $W_{0} \Subset U \backslash I_{f}$. Now it is clear that $\Gamma_{f_{k}} \cap\left(W_{0} \times X\right)$ cannot approach $\Gamma_{f} \cap\left(W_{0} \times X\right)=W$ with multiplicity more than one, because $\Gamma_{f_{k}}$ is a graph of a holomorphic map over $W_{0}$ for $k \gg 1$.

We proved that every irreducible branch of $\Gamma_{f} \cap V$ the graphs $\Gamma_{f_{k}}$ approach with multiplicity one. Therefore $j\left(\Gamma_{f_{k}} \cap V\right) \rightarrow \Delta^{n}$ is a ramified covering of the same degree $d$ as $j\left(\Gamma_{f} \cap V\right) \rightarrow \Delta^{n}$ for $k \gg 1$. This proves at a time that $\Gamma_{f_{k}}$ converge to $\Gamma_{f}$ in the topology of cycles and that their volumes are uniformly bounded.

Strong convergence has some nice features, one was mentioned in the Introduction. Moreover, as it is explained in [20], strong topology is natural in studying fix points of meromorphic self-mappings of compact complex manifolds. But domains of strong convergence and strong normality are quite arbitrary. We shall explain this in more details. Let $\mathcal{F}$ be a family of meromorphic mappings from a complex manifold $U$ to a disk convex complex manifold $X$.

Definition 2.7. The set of normality of $\mathcal{F}$ is the maximal open subset $\mathcal{N}_{\mathcal{F}}$ of $U$ such that $\mathcal{F}$ is relatively compact on $\mathcal{N}_{\mathcal{F}}$. If $\mathcal{F}=\left\{f_{k}\right\}$ is a sequence then the set of convergence of $\mathcal{F}$ is the maximal open subset of $U$ such that $f_{k}$ converge on compacts of this subset.

To be relatively compact in this definition means that from every sequence of elements of $\mathcal{F}$ one can extract a converging on compacts subsequence. The sense of convergence (strong, weak or other) should be each time specified.

\section{Example 2.8.}

1. Let $X$ be a Hopf three-fold $X:=\mathbb{C}^{3} \backslash\{0\} / z \sim 2 z$. Denote by $\pi: \mathbb{C}^{3} \backslash\{0\} \rightarrow X$ the canonical projection. Let $D \Subset \mathbb{C}^{2}$ be any bounded domain. Take a sequence $\left\{a_{n}\right\} \subset D$ accumulating to every point on $\partial D$. Let $g_{n}: \mathbb{C}^{2} \rightarrow \mathbb{C}^{3}$ be defined as $g_{n}(z)=\left(z-a_{n}, 1 / n\right)$. Set $f_{n}:=\pi \circ g_{n}$. Then the set of normality of $\mathcal{F}=\left\{f_{n}\right\}$ has $D$ as one of its connected components.

Remark 2.9. For an analogous example with $X$ projective see [18, Example 4].

2. The same example is instructive when understanding the notion of weak convergence. Take a converging to zero sequence $a_{n}$. Then $f_{n}$ from this example will converge on compacts of $\mathbb{C}^{2} \backslash\{0\}$ but the limit will not extend to zero meromorphically. I.e., $f_{n}$ will not converge weakly in any neighborhood of the origin. 


\section{Pseudoconvexity of sets of normality}

\subsection{Weak convergence and proof of Theorem 1.5}

In view of such examples a weaker notion of convergence for meromorphic mappings was introduced in [18]. Let $f \in \mathcal{M}(U, X)$ be a meromorphic map from $U$ to $X$ and let $\left\{f_{k}\right\} \subset \mathcal{M}(U, X)$ be a sequence of meromorphic mappings.

Definition 3.1. We say that $f_{k}$ converge weakly to $f$ ( $w$-converge) if there exists an analytic subset $A$ in $U$ of codimension at least two such that $f_{k}$ converge strongly to $f$ on $U \backslash A$.

Remark 3.2. $f_{k}$ converge weakly to $f$ if and only if for every compact of $U \backslash I_{f}$ all $f_{k}$ are holomorphic in a neighborhood of this compact for $k$ big enough and uniformly converge there to $f$ as holomorphic mappings. Indeed, let $A$ be the minimal analytic set of codimension $\geq 2$ such that $f_{k}$ converge strongly to $f$ on $U \backslash A$. Then $A$ must be contained in $I_{f}$ because if there exists a point $a \in A \backslash I_{f}$ then $f$ is holomorphic in some neighborhood $V \ni a$ and then, by Rouché Principle of [18] $f_{k}$ for $k \gg 1$ are holomorphic on compacts in $V \backslash A$ and converge uniformly (on compacts) to $f$ there. From here and the fact that $\operatorname{codim} A \geq 2$ one easily gets that $f_{k}$ are holomorphic on compacts in $V$ and converge to $f$.

Now let us turn to the sets of weak convergence/normality. Sets of strong normality obviously are well defined, i.e., they do exist. The existence of sets of weak normality was proved in [18, Corollary 1.2.1 a].

Remark 3.3. In the formulation of this Corollary the Author of [18] speaks about "weak convergence" but the proof is about "weak normality".

Domains of weak convergence of meromorphic mappings turn to be pseudoconvex for a large class of target manifolds. This follows from the "mutual propagation principle" stated in Theorem 1.5 in the Introduction. Let us give a proof of it.

Proof of Theorem 1.5. Let us prove the part (b) first.

Step 1. Extension of the limit. First of all by the main result of [19] every meromorphic map $f: U \rightarrow X$ extends to a meromorphic map $f: \hat{U} \backslash A \rightarrow X$, where $A$ is closed, complete $(n-2)$-polar subset of $\hat{U}$ of Hausdorff $(2 n-3)$-measure zero. In more details that means that for every point $a \in A$ there exists a coordinate neighborhood $V \cong \Delta^{n-2} \times \mathbb{B}^{2}$ of $a=0$ such that $A \cap\left(\Delta^{n-2} \times \partial \mathbb{B}^{2}\right)=\emptyset$ and for every $z^{\prime} \in \Delta^{n-2}$ the intersection $A_{z^{\prime}}:=A \cap \mathbb{B}_{z^{\prime}}^{2}$ is a zero dimensional complete pluripolar subset of $\mathbb{B}_{z^{\prime}}^{2}:=\left\{z^{\prime}\right\} \times \mathbb{B}^{2}$. Here $\mathbb{B}^{2}$ stands for the unit ball in $\mathbb{C}^{2}$. Moreover, if $A \neq \emptyset$ then $f\left(\mathbb{S}_{z^{\prime}}^{3}\right)$ is not homologous to zero in $X$. Here $\mathbb{S}_{z^{\prime}}^{3}=\partial \mathbb{B}_{z^{\prime}}$ is the standard three-dimensional sphere in $\mathbb{C}^{2}$.

Let $U^{\prime}$ be the maximal open subset of $\hat{U} \backslash\left(I_{f} \cup A\right)$ such that $f_{k}$ converge to $f$ on compacts on $U^{\prime}$ as holomorphic mappings. 
Step 2. $U^{\prime}$ is locally pseudoconvex in $\hat{U} \backslash\left(I_{f} \cup A\right)$. If not then by DocquierGrauert criterion, see [7], there would exist a point $b \in \partial U^{\prime} \backslash\left(I_{f} \cup A\right)$ and a Hartogs figure $h: H_{\varepsilon}^{n} \rightarrow U^{\prime}$ imbedded to $U^{\prime}$ such that the image $h\left(\Delta^{n}\right)$ of the corresponding polydisk contains $b$. All this is local and therefore we can assume that $h\left(\Delta^{n}\right)$ is relatively compact in $U \backslash\left(I_{f} \cup A\right)$. Pulling back $f_{k}$ and $f$ to $\Delta^{n}$ we arrive to contradiction as follows. By the Theorem of Siu, see [40], there exists a Stein neighborhood $V$ of the graph of $f \circ h$ in $\Delta^{n} \times X$. Since for every compact $K \Subset H_{\varepsilon}^{n}$ we have that the graph of $\left.f_{k}\right|_{K}$ is contained in $V$ we conclude the same for every compact of $\Delta^{n}$. Now $f_{k} \circ h$ converge to $f \circ h$ on compacts in $\Delta^{n}$ as holomorphic mappings. But that mean that they converge also around the preimage of $b$. Contradiction. Since $\hat{U}$ was supposed to be the envelope of holomorphy of $U$ we obtain that $U^{\prime}=\hat{U} \backslash\left(I_{f} \cup A\right)$.

Step 3. Removing A. Suppose $A$ is non-empty. Take a sphere $\mathbb{S}_{z^{\prime}}^{3}$ as described in Step 1 for some point $a \in A$. Using the fact that $I_{f}$ is of codimension $\geq 2$ we can take $\mathbb{S}_{z^{\prime}}^{3}$ not to intersect $I_{f}$ as well. I.e., $\mathbb{S}_{z^{\prime}}^{3} \subset U^{\prime} . f_{k}\left(\mathbb{S}_{z^{\prime}}^{3}\right)$ is homologous to zero in $X$, because $f_{k}$ meromorphically extends to the corresponding $\mathbb{B}_{z^{\prime}}^{2}$. Moreover $f_{k}$ converge to $f$ in a neighborhood of $\mathbb{S}_{z^{\prime}}^{3}$. This implies that $f\left(\mathbb{S}_{z^{\prime}}^{3}\right)$ is also homologous to zero and therefore $A$ should be empty. Contradiction. Part (b) is proved.

The proof of (a) is a particular case of the Step 2 of the proof of part (b).

Remark 3.4. We gave a proof of Theorem 1.5 here because the proof of an analogous statement in [18] uses a stronger extension claim from the subsequent paper [19]. Namely the Author claimed that $A$ appearing in the Step 1 of the proof is analytic of codimension two. This was not achieved in [19] (and is not clear for us up to know). Therefore we find necessary to remark that vanishing of $(2 n-3)$ dimensional measure of $A$ together with homological characterization of the obstructions for the meromorphic extension is, in fact, sufficient for our particular task here.

\subsection{Gamma convergence of meromorphic mappings}

Let again $f_{k}$ be a sequence of meromorphic mappings between complex manifolds $U$ and $X$, the last is supposed to be disk-convex. Let $f \in \mathcal{M}(U, X)$ be a meromorphic map.

Definition 3.5. We say that $f_{k} \Gamma$-converge to $f$ if:

i) there exists an analytic subset $A \subset U$ such that $f_{k}$ strongly converge to $f$ on $U \backslash A$

ii) for every divisor $H$ in $X$, such that $f(U) \not \subset H$ and every compact $K \Subset U$ the volumes of $f_{k}^{*} H \cap K$ counted with multiplicities are uniformly bounded for $k \gg 1$.

Remark 3.6. This notion is strictly weaker than the weak convergence because $A$ can have components of codimension one, and remark that the item (i) is automatically satisfied by a weakly converging sequence, because divisors $f^{*} H$ extend from 
$U \backslash A$ to $U$ and if they have bounded volume on compacts of $U \backslash A$ then the same is true on compacts of $U$. All this obviously follows from the ingredients involved in the proof of Bishop's compactness theorem, see [5] or [43]. It might be convenient to add to $A$ the indeterminacy set of $f$ and then, see Remark $3.2, f_{k}$ will converge to $f$ uniformly on compacts of $U \backslash A$ as holomorphic mappings.

\section{Example 3.7.}

a) Consider the following sequence of holomorphic mappings $f_{k}: \Delta \rightarrow \mathbb{P}^{1}$ :

$$
f_{k}: z \rightarrow\left[1: 1+\frac{1}{z}+\ldots+\frac{1}{z^{k} k !}\right]=\left[z^{k}: z^{k}+z^{k-1}+\ldots+\frac{1}{k !}\right]
$$

It is clear that $f_{k}$ converges on compacts of $\Delta \backslash\{0\}$ to $f(z)=\left[1: e^{\frac{1}{z}}\right]$ but, as it is clear from the second expression in (3.1) the preimage counting with multiplicities of the divisor $H=\left\{Z_{0}=0\right\}$ is $k[0]$ (here $\left[Z_{0}: Z_{1}\right]$ are homogeneous coordinates in $\left.\mathbb{P}^{1}\right)$, i.e., has unbounded volume. And indeed, this sequence should not be considered as converging one, because its limit is not holomorphic on $\Delta$.

b) Set $f_{k}(z)=\left[z: z-\frac{1}{k}\right]: \Delta \rightarrow \mathbb{P}^{1}$. This sequence clearly converges to the constant map $f(z)=[z: z]=[1: 1]$ on compacts of $\Delta \backslash\{0\}$. Moreover, the preimage of any divisor $H=\left\{P\left(z_{0}, z_{1}\right)=0\right\}$ under $f_{k}$ is $\left\{z \in \Delta: P\left(z, z-\frac{1}{k}\right)=\right.$ $0\}$, i.e., is a set of points, uniformly bounded in number counting with multiplicities. Therefore this sequence $\Gamma$-converge (but does not converge weakly).

Example 3.8. Consider the following sequence of meromorphic functions on $\Delta^{2}$ (i.e., meromorphic mappings to $\mathbb{P}^{1}$ ):

$$
f_{k}\left(z_{1}, z_{2}\right)=\left[z_{1}: 2^{-k} z_{2}^{k}\right]
$$

The limit map is constant $f(z)=[1: 0]$. $f_{k}$ converge to $f$ strongly (uniformly in fact) on compacts of $\Delta^{2} \backslash\left\{z_{1}=0\right\}$. If $\left[Z_{0}: Z_{1}\right]$ are homogeneous coordinates in $\mathbb{P}^{1}$ then the preimage of the divisor $\left[Z_{1}=0\right]$ is $k\left[z_{2}=0\right]$, i.e., this sequence does not converge even in $\Gamma$-sense on $\Delta^{2}$.

Remark 3.9. Examples 3.7 (a) and 3.8 are examples of converging outside of an analytic set of codimension one sequences which are not $\Gamma$-converging. In the first case the limit does not extend to the whole source, in the second it does. Convergence of meromorphic mappings of this type was introduced and studied by Rutishauser in [37].

If in Definition 2.7 the underlying convergence is $\Gamma$-convergence we get the corresponding notions of a convergence/normality set. Let us conclude this general discussion with the following

Proposition 3.10. Let $X$ be a disk-convex Gauduchon manifold. Then the sets of $\Gamma$-convergence/normality of meromorphic mappings with values in $X$ are locally pseudoconvex. 
Proof. We shall prove the statement for the sets of $\Gamma$-normality, the case of sets of convergence obviously follows. Let $D$ be the maximal open subset of $U$ where the family $\mathcal{F}$ is $\Gamma$-normal. Suppose that $D$ is not pseudoconvex. Then by DocquierGrauert criterion, see [7], there exists an imbedding $h: H_{\varepsilon}^{n} \rightarrow D$ of a Hartogs figure into $D$ such that $h$ extends to an immersion of the polydisk to $U$ with $h\left(\Delta^{n}\right) \cap$ $\partial D \neq \emptyset$. Recall that Hartogs figure is the following domain

$$
H_{\varepsilon}^{n}:=\left(\Delta_{\varepsilon}^{n-1} \times \Delta\right) \cup\left(\Delta^{n-1} \times A_{1-\varepsilon, 1}\right)
$$

where $A_{1-\varepsilon, 1}:=\Delta \backslash \bar{\Delta}_{1-\varepsilon}$ is an annulus. Let us pull-back our family to $\Delta^{n}$ by $h$ and therefore without loss of generality we can suppose that $U=\Delta^{n}, \mathcal{F}$ is a family of meromorphic mappings from $\Delta^{n}$ to $X, H_{\varepsilon}^{n} \subset D \subset \Delta^{n}$ is the set of $\Gamma$-normality of $\mathcal{F}$ such that $D \neq \Delta^{n}$.

That means that there exists a sequence $\left\{f_{k}\right\} \subset \mathcal{F}$, which converges on $D$ but does not not $\Gamma$-converge on compacts in $\Delta^{n}$. Let us see that this is impossible. Let $f: D \rightarrow X$ be the $\Gamma$-limit of $f_{k}$. Denote by $A$ the analytic set in $D$ such that $f_{k}$ converge to $f$ on compacts of $D \backslash A$. By [19] $f$ extends to $\Delta^{n} \backslash S$, where $S$ is closed $(n-2)$-complete polar subset of $\Delta^{n}$. Let $A^{\prime}$ be the pure $(n-1)$-dimensional part of $A$. By the theorem of Grauert we have two cases.

Case 1. The envelope of holomorphy of $D \backslash A^{\prime}$ is $\Delta^{n}$. In that case the Theorem 1.5 is applicable with $U=D \backslash A^{\prime}$ and $\hat{U}=\Delta^{n}$ and gives us the weak (and therefore $\Gamma)$ convergence of $f_{k}$ on $\Delta^{n}$.

Case 2. $A^{\prime}$ extends to a hypersurface $\tilde{A}$ in $\Delta^{n}$ and $\Delta^{n} \backslash \tilde{A}$ is the envelope of holomorphy of $D \backslash A^{\prime}$. In that case again by Theorem $1.5 f_{k}$ weakly converge to $f$ on $\Delta^{n} \backslash \tilde{A} . S \backslash \tilde{A}$ is removable for $f$, see the Step 3 in the proof of Theorem 1.5. Therefore $f_{k}$ strongly converge to $f$ outside of a proper analytic set $A \cup I_{f}$. We need now to prove that $f$ is extendable to $\Delta^{n}$, i.e., that $S$ is empty. By Lemma 7.3 below the areas of disks $f_{k}\left(\Delta_{z^{\prime}}\right)$ are bounded uniformly on $k$ and on $z^{\prime} \in \Delta^{n-1}(1-\varepsilon)$ for any fixed $\varepsilon>0$, here $\Delta_{z^{\prime}}:=\left\{z^{\prime}\right\} \times \Delta$. Therefore the areas of $f\left(\Delta_{z^{\prime}}\right)$ are bounded to. Theorem 1.5 together with Proposition 1.9 from [19] imply now that $f$ meromorphically extends onto $\Delta^{n-1}(1-\varepsilon) \times \Delta$. Therefore it extends to $\Delta^{n}$. The condition i) of Definition 3.5 is fulfilled.

Let $H$ be a divisor in $X$. Then for every compact $K \Subset H_{\varepsilon}^{n}$ the volumes of $f_{k}^{*} H \cap K$ counted with multiplicities are bounded. By Oka-Riemenschneider theorem, see [36], the volumes of the extensions of these divisors are bounded on compacts of $\Delta^{n}$ too. This verifies the condition ii) of Definition 3.5. Proposition is proved.

\section{Convergence of mappings with values in projective space}

Now let us examine our notions of convergence on the example when the target manifold is a complex projective space. 


\subsection{Meromorphic mappings to complex projective space}

Let a meromorphic mapping $f: U \rightarrow \mathbb{P}^{N}$ be given. Without loss of generality we suppose that the image of $f$ is not contained in a hyperplane. Then the (complete) inverse image $f^{-1}(H)$ under $f$ of a hyperplane $H$ is a divisor in $U$. By $f^{-1}\left(\mathbb{P}^{n} \backslash H\right)$ we shall understand $U \backslash f^{-1}(H)$. Denote by $\left[w_{0}: w_{1}: \ldots: w_{N}\right]$ the homogeneous coordinates of $\mathbb{P}^{N}$. Let $U_{j}=\left\{w \in \mathbb{P}^{N}: w_{j} \neq 0\right\}$ and let $\frac{w_{0}}{w_{j}}, \ldots, \frac{w_{N}}{w_{j}}$ be affine coordinates in $U_{j}$. Set $D_{j}:=f^{-1}\left(U_{j}\right)$, i.e., $D_{j}=U \backslash f^{-1}\left(H_{j}\right)$, where $H_{j}:=\left\{w_{j}=0\right\}$. Since $U_{0}$ is isomorphic to $\mathbb{C}^{N}$ the restriction $\left.f\right|_{D_{0}}: D_{0} \longrightarrow U_{0}$ is given by holomorphic functions $\frac{w_{1}}{w_{0}}=f_{1}(z), \ldots, \frac{w_{N}}{w_{0}}=f_{N}(z)$. The coordinate change in $\mathbb{P}^{N}$ shows that $\left.f\right|_{D_{0} \cap D_{j}}: D_{0} \cap D_{j} \longrightarrow \mathbb{P}^{N}$ is given by functions $\frac{w_{1}}{w_{0}}=\frac{1}{f_{j}(z)}, \ldots, \frac{w_{N}}{w_{0}}=\frac{f_{N}(z)}{f_{j}(z)}$ which are holomorphic in $D_{j}$. Therefore functions $f_{1}, \ldots, f_{N}$ are meromorphic on $D_{0} \cup D_{j}$. This proves that $f_{1}, \ldots, f_{N}$ are meromorphic on $\bigcup_{j=0}^{N} D_{j} \subset U$. We have that $U \backslash \bigcup_{j=0}^{N} D_{j}=\bigcap_{j=0}^{N} f^{-1}\left(H_{j}\right)$, i.e., for every point from this set the image of every its neighborhood intersects every $H_{j}$. Such point can be only an indeterminacy point of $f$. I.e., $U \backslash \bigcup_{j=0}^{N} D_{j} \subset I_{f} . I_{f}$ is analytic of codimension $\geq 2$ and therefore by the theorem of Levi, see [29] or [14], every $f_{j}$ meromorphically extends to $U$.

If $f_{1} \equiv \ldots \equiv f_{n} \equiv 0$ then $f(U) \equiv 0 \in U_{0}$. If not, let $f_{1} \not \equiv 0$. One finds holomorphic functions $h_{j}$ et $g_{j} 0 \leq j \leq N$ in a polydisk neighborhood $V$ of a given point $x \in U, g_{j} \neq 0$ such that

$$
f_{1}=\frac{h_{1}}{g_{1}}, \ldots, f_{N}=\frac{h_{N}}{g_{N}}
$$

and therefore gets

$$
f:=\left[1: \frac{h_{1}}{g_{1}}: \ldots: \frac{h_{N}}{g_{N}}\right]=\left[\prod_{j=1}^{N} g_{j}: h_{1} \prod_{j=2}^{N} g_{j}: \ldots: h_{N} \prod_{j=1}^{N-1} g_{j}\right] .
$$

This proves that $f$ can be locally written in the form

$$
f(z):=\left[f_{0}(z): f_{1}(z): \ldots: f_{N}(z)\right]
$$

as claimed.

\subsection{Weak convergence of mappings with values in projective space}

Let us prove now the part ii) of Theorem 1.3 from the Introduction. I.e.,

Proposition 4.1. A sequence of meromorphic mappings $f_{k}$ from a complex manifold $U$ to $\mathbb{P}^{N}$ converges weakly on compacts of $U$ if an only if for every point $z_{0} \in U$ there exists a neighborhood $V \ni z_{0}$ and reduced representations $f_{k}=\left[f_{k}^{0}: \ldots\right.$ : $\left.f_{k}^{N}\right], f=\left[f^{0}: \ldots: f^{N}\right]$ in $V$ such that for every $0 \leq j \leq N f_{k}^{j}$ converge to $f^{j}$ uniformly on $V$. 
$\Rightarrow$ Let $f_{k} \rightarrow f$, i.e., $f_{k}$ converge to $f$ weakly. Shrinking $U$ we suppose that all $f_{k}$ and $f$ admit reduced representations

$$
f_{k}=\left[f_{k}^{0}: \ldots: f_{k}^{N}\right]
$$

and

$$
f=\left[f^{0}: \ldots: f^{N}\right]
$$

correspondingly. Up to making a linear coordinate change in $\mathbb{P}^{N}$ we can suppose that $f[U]$ is not contained in any of coordinate hyperplanes, i.e., that $f^{j} \not \equiv 0$ for all $0 \leq j \leq N$. Set

$$
Z^{j}=\left\{z \in U: f^{j}(z)=0\right\},
$$

and note that $\bigcap_{j=1}^{N} Z^{j}=I_{f}$. Since $f_{k}$ converge on compacts in $U_{j}:=U \backslash Z^{j}$ to $f$, see Remark 3.2, we see, taking $j=0$, that

$$
\frac{f_{k}^{j}}{f_{k}^{0}} \rightrightarrows \frac{f^{j}}{f^{0}}
$$

for all $j$ on compacts in $U_{0}$. Denote by $Z_{k}^{0}$ the zero divisors of $f_{k}^{0}$ and note that they leave every compact of $U_{0}$ as $n \rightarrow \infty$.

Lemma 4.2. Divisors $Z_{k}^{0}$ converge to $Z^{0}$ in cycle space topology.

Let us prove this lemma first. Fix a point $a \in Z^{0} \backslash Z^{j}$ (if $Z^{0} \backslash Z^{j}$ is not empty) and take a relatively compact neighborhood $V \ni a$ such that $\bar{V} \cap Z^{j}=\emptyset$. We have that $f_{k}^{0} / f_{k}^{j} \rightrightarrows f^{0} / f^{j}$ on $\bar{V}$. The Rouché's theorem easily implies now that $Z_{k}^{0} \cap V$ converge to $Z^{0} \cap V$ as currents.

Remark 4.3. In fact the cycle space topology on the space of divisors coincides with the topology of uniform convergence of defining them holomorphic functions, see [42]. And this immediately gives the previous assertion.

We conclude from here that $Z_{k}^{0}$ converge to $Z^{0}$ as cycles on compacts in $U \backslash I_{f}$. But then by [33], Theorem II, we obtain that they converge on the whole of $U$. Lemma 4.2 is proved.

We continue the proof of the Theorem. Shrinking $U$ if necessary we can suppose that $U$ is biholomorphic to $\Delta^{n}=\Delta^{n-1} \times \Delta$ and $Z_{k}^{0} \cap U$ regularly covers $\Delta^{n-1}$ for $k \gg 1$. Now each $Z_{k}^{0}$ can be written as the zero set of a uniquely defined unitary polynomial $P_{k}$ from $\mathcal{O}_{\Delta^{n-1}}\left[z_{n}\right]$ and these $P_{k}$ uniformly converge to $P$ - the defining polynomial for $Z^{0}$. After multiplying each $\left[f_{k}^{0}: \ldots: f_{k}^{N}\right]$ by the unit $P_{k} / f_{k}^{0}$ we get the reduced representations

$$
f_{k}=\left[P_{k}: g_{k}^{1} \ldots: g_{k}^{N}\right] .
$$


The same with

$$
f=\left[P: g^{1} \ldots: g^{N}\right] .
$$

But now $P_{k} \rightrightarrows P$ and therefore from (4.4), which reads now as

$$
\frac{g_{k}^{j}}{P_{k}} \rightrightarrows \frac{g^{j}}{P}
$$

on compacts in $U_{0}$, we get that for every $1 \leq j \leq N g_{k}^{j} \rightrightarrows g^{j}$ on compacts in $U_{0}=U \backslash Z^{0}$. But from the maximum principle it follows that $g_{k}^{j} \rightrightarrows g^{j}$ on compacts in $U$.

$\Leftarrow$ For proving the inverse statement we start with converging reduced representations (4.2) to (4.3), i.e., $f_{k}^{j} \rightrightarrows f^{j}$ on $U$. Then for every $0 \leq j \leq N$ on every $U_{j}=U \backslash Z^{j}$ we get a convergence on compacts

$$
\left(\frac{f_{k}^{0}}{f_{k}^{j}}, \ldots, \frac{f_{k}^{N}}{f_{k}^{j}}\right) \rightrightarrows\left(\frac{f^{0}}{f^{j}}, \ldots, \frac{f^{N}}{f^{j}}\right) .
$$

And since the codimension of $I_{f}=\bigcap Z^{j}$ is at least two we deduce the weak convergence of $f_{k}$ to $f$.

\subsection{Strong convergence and convergence of meromorphic functions}

Strong convergence of meromorphic maps into $\mathbb{P}^{N}$ can be described in the following way. First, if $f_{k} \rightarrow f$ then $f_{k} \rightarrow f$. Therefore $\left[f_{k}^{0}: \ldots: f_{k}^{N}\right] \rightrightarrows\left[f^{0}: \ldots: f^{N}\right]$ for an appropriate reduced representations. According to (2.2) the volume of the graph of $f_{k}$ is

$$
\int_{U \backslash I_{f_{k}}}\left(\omega_{1}+f_{k}^{*} \omega_{F S}\right)^{n}=\int_{U \backslash I_{f}} \sum_{j=0}^{n} C_{n}^{j} \omega_{1}^{j} \wedge f_{k}^{*} \omega_{F S}^{n-j} .
$$

Since $f_{k}^{*} \omega_{F S}=d d^{c} \ln \left\|f_{k}\right\|^{2}$ this is nothing but the non-pluripolar Monge-Ampère mass of $\ln \left\|f_{k}\right\|^{2}$ as appeared in (1.3). By Proposition 1.1 volumes of $\Gamma_{f_{k}}$ converge to the volume of $\Gamma_{f}$, i.e.,

$$
\int_{U \backslash I_{f_{k}}} \omega_{1}^{j} \wedge\left(d d^{c} \ln \left\|f_{k}\right\|^{2}\right)^{n-j} \rightarrow \int_{U \backslash I_{f}} \omega_{1}^{j} \wedge\left(d d^{c} \ln \|f\|^{2}\right)^{n-j}
$$

for $0 \leq j<n$. In the case $U=\Delta^{n}$ this gives (1.3). Vice versa, if one has convergence of volumes the appearance of an exceptional component is impossible and we conclude the part (ii of Theorem 1.3: 
Proposition 4.4. $f_{k}$ converge to $f$ strongly if and only if

i) the appropriate reduced representations converge uniformly;

ii) for every $0 \leq j \leq n-1$ one has (4.7).

Now let us descend to the convergence of meromorphic functions. Meromorphic functions on a complex manifold $U$ are exactly the meromorphic mappings from $U$ to $\mathbb{P}^{1}$. I.e., all our previous results and notions are applicable to this case.

Proposition 4.5. If a sequence $\left\{f_{k}\right\}$ of meromorphic functions converges weakly then it converges strongly.

Proof. Let $f$ be the weak limit of $f_{k}$. We shall see in a moment, see Corollary 5.6 that volumes of graphs in this case are uniformly bounded over compacts in $U$. Therefore after going to a subsequence we get that the Hausdorff $\operatorname{limit} \hat{\Gamma}:=\lim \Gamma_{f_{k}}$ is a purely $n$-dimensional analytic subset of $U \times \mathbb{P}^{1}$. We claim that $\lim \Gamma_{f_{k}}=\Gamma_{f}$ in fact, i.e., that there are no exceptional components. If not take any irreducible component $\Gamma$ of this limit different from $\Gamma_{f}$. Denote by $\gamma$ its projection to $U . \gamma$ is a proper analytic set of codimension at least two $U$. But then $\Gamma$ should be contained in $\gamma \times \mathbb{P}^{1}$ and the last analytic set is of dimension $\operatorname{dim} U-1$. This is impossible, because all components of $\lim \Gamma_{f_{k}}$ are of pure dimension $\operatorname{dim} U$. Therefore $\gamma=\emptyset$ and $\lim \Gamma_{f_{k}}=\Gamma_{f}$.

\subsection{Gamma convergence in projective case}

In [10] and subsequent papers of Fujimoto the following type of convergence of meromorphic mappings with values in $\mathbb{P}^{N}$ was considered, it was called the $m$ convergence (or meromorphic convergence): $f_{k} m$-converge to $f$ if there exist reduced (admissible in the terminology of [10]) representations $f_{k}=\left[f_{k}^{0}: \ldots: f_{k}^{N}\right]$ which converge uniformly on compacts to $f=\left[f^{0}: \ldots: f^{N}\right]$, but the last is not supposed to be reduced (i.e., admissible), only not all $f^{j}$ are identically zero. Let us prove the item i) of Theorem 1.3.

Proposition 4.6. When the target manifold $X$ is the complex projective space $\mathbb{P}^{N}$ the $\Gamma$-convergence of meromorphic mappings is equivalent to $m$-convergence in the sense of Fujimoto.

Proof. $\Rightarrow$ Suppose that $f_{k} \stackrel{\Gamma}{\rightarrow} f$. Let $\gamma$ be the an analytic subset of $U$ such that our sequence converge strongly on compacts of $U \backslash \gamma$. We add to $\gamma$ also the indeterminacies of the limit $f$ and therefore $f_{k}$ will converge to $f$ on $U \backslash \gamma$ in compact open topology. Let $f=\left[f^{0}: \ldots: f^{N}\right]$ be some reduced representation of the limit map. Making linear change of coordinates we can suppose, without loss of generality that $f^{0} \not \equiv 0$, i.e., that $f(U) \not \subset H_{0}$, where $H_{0}=\left\{Z_{0}=0\right\}$ in homogeneous coordinates $\left[Z_{0}: \ldots: Z_{N}\right]$ of $\mathbb{P}^{N}$. We have that $f_{k}^{*} H_{0}$ converge on compacts in $U$ in the cycle space topology (after taking a subsequence). 
Take some $a \in \gamma$ and choose a chart $(V, j)$ adapted both to $\gamma$ and $f^{*} H_{0}$ with coordinates $z_{1}, \ldots, z_{n}$ around $a$ in such a way that $a=0$ and $\left(\gamma \cup f^{*} H_{0}\right) \cap$ $\left(\Delta^{n-1} \times \Delta\right)$ projects to $\Delta^{n-1}$ properly. Then $f_{k}^{*} H_{0} \cap V$ also projects to $\Delta^{n-1}$ properly for $k \gg 1$. After going to a subsequence once more we can fix the degree $d$ of ramified coverings $f_{k}^{*} H_{0} \cap V \rightarrow \Delta^{n-1}$ and write the corresponding polynomials $P_{k} \in \mathcal{O}_{\Delta^{n-1}}\left[z_{n}\right]$ defining $f_{k}^{*} H_{0} \cap V . P_{k}$ converge to some $P$ on (compacts of) $\Delta^{n-1}$. Let $f_{k}=\left[\begin{array}{lll}f_{k}^{0}: \ldots: f_{k}^{N}\end{array}\right]$ be some reduced representations of $f_{k}$ on $V$. Notice that $f_{k}^{*} H_{0} \cap V=\left\{f_{k}^{0}=0\right\}$. Divide each such representation by the unit $f_{k}^{0} / P_{k}$ and get representations $f_{k}=\left[P_{k}: g_{k}^{1}: \ldots: g_{k}^{N}\right]$ with converging first terms $P_{k}$. At the same time $\left(g_{k}^{1} / P_{k}, \ldots, g_{k}^{N} / P_{k}\right)$ represents $f_{k}$ in nonhomogeneous coordinates of the chart $Z_{0} \neq 0$ on $\mathbb{P}^{N}$. Therefore $g_{k}^{j} / P_{k}$ converge to some $f^{j}$ on compacts of $V \backslash\left(\gamma \cup f^{*} H_{0}\right)$. Therefore $g_{k}^{j}$ converge to $g^{j}:=f^{j} P$ on compacts of $V \backslash\left(\gamma \cup f^{*} H_{0}\right)$ to. By maximum principle they converge everywhere on $V$ to the extension of $g^{j}$. We get that reduced representations $f_{k}=\left[P_{k}: g_{k}^{1}: \ldots: g_{k}^{N}\right]$ converge term by term to a (may be non reduced) representation $\left[P: g^{1}: \ldots: g^{N}\right]$ and this can be only a representation of $f$.

$\Leftarrow$ Suppose now that $f_{k} m$-converge to $f$. Again change coordinates in $\mathbb{P}^{N}$, if necessary, in such a way that $f(U) \not \subset H_{0}$. Let $V$ be a neighborhood of some point $a \in U$. If $a \in f^{*} H_{0}$ then take $(V, j)$ to be an adapted chart to this divisor. In any case take $V$ to be biholomorphic to $\Delta^{n-1} \times \Delta$. Let $F_{k}=\left(f_{k}^{0}, \ldots, f_{k}^{N}\right)$ be the lifts of $f_{k}$ to $\mathbb{C}^{N+1}$ in $V$ such that $F_{k}$ converge to the lift $F=\left(f^{0}, \ldots, f^{N}\right)$ of $f$. From here one gets immediately that $f_{k}^{j} / f_{k}^{0}$ converge to $f^{j} / f^{0}$ uniformly on compacts of $V \backslash\left\{f^{0}=0\right\}$, i.e., that our maps converge strongly outside of a divisor.

Now let $H=\left\{P\left(Z_{0}, \ldots, Z_{N}\right)=0\right\}$ be a divisor such that $f(U) \not \subset H$. Using convergence of lifts $F_{k}=\left(f_{k}^{0}, \ldots, f_{k}^{N}\right)$ to $F=\left(f^{0}, \ldots, f^{N}\right)$ one gets that $f_{k}(U) \not \subset H$ for $k \gg 1$. One has also that $P\left(f_{k}^{0}, \ldots, f_{k}^{N}\right)$ uniformly converge to $P\left(f^{0}, \ldots, f^{N}\right)$ and this is equivalent to the convergence of divisors.

Remark 4.7. The relation between weak/gamma convergence and $m$-convergence for the case of $X=\mathbb{P}^{N}$ was indicated without proof in [18].

\section{Bloch-Montel type normality criterion}

The aim of this section is to test the notion of weak convergence on the BlochMontel type normality statement, i.e., we are going to prove here the Theorem 1.8 from the Introduction.

\subsection{Preliminaries}

Before proceeding with the proof let us recall few basic facts. We start with an extended version of Zalcman's lemma, see [31]: 
Lemma 5.1. A family $\mathcal{F}$ of holomorphic mappings from $\Delta^{n}$ to a compact Hermitian manifold $(X, h)$ is not normal at $z_{0} \in \Delta^{n}$ if and only if there exist sequences $z_{k} \rightarrow z_{0}, r_{k} \searrow 0, f_{k} \in \mathcal{F}$ such that $f_{k}\left(z_{k}+r_{k} w\right)$ converge uniformly on compacts in $\mathbb{C}^{n}$ to a non-constant entire mapping $f: \mathbb{C}^{n} \rightarrow X$ such that $\|d f(w)\|_{h} \leq 2$ for all $w \in \mathbb{C}^{n}$.

This $f$ may well have rank one. We shall also need the following result from [21], which is a precise version of Gromov compactness theorem (we shall need it in the integrable case only):

Proposition 5.2. Let $u_{k}: \Delta \rightarrow X$ be a sequence of holomorphic maps into a diskconvex Hermitian manifold $(X, h)$ with uniformly bounded areas, which uniformly converges on some annulus $A_{1-\varepsilon, 1}$ adjacent to the boundary $\partial \Delta$. Then $u_{k}$ converge to stable complex curve over $X$ after a reparametrization. Moreover, the compact components of the limit are rational curves.

For the notions of stable curve over $X$, convergence after a reparametrization, as well as for the proof we refer to [21]. The obvious conclusion from this type of convergence is the following:

Corollary 5.3. If $u_{k}$ converge in stable sense to $u$ and $u(\Delta)$ intersects a divisor $H$ in $X$, but is not contained in $H$, then all $u_{k}(\Delta)$ intersect $H$ for $k \gg 1$.

Proof. It was proved in [22] (more details are given in [21]) that for any $k \gg 1$ one can join $u_{k}$ with $u$ by a holomorphic one parameter family of stable maps, see [22, Proposition 2.1.3 ] for the exact statement. For us it is sufficient to understand that there exists a normal complex surface $Y \stackrel{\pi}{\rightarrow} \Delta$ foliated over the disk $\Delta$ such that all fibers $Y_{s}:=\pi^{-1}(s)$ are disks and a holomorphic mapping $\mathcal{U}: Y \rightarrow X$ such that $\left.\mathcal{U}\right|_{Y_{0}}=u$ and $\left.\mathcal{U}\right|_{Y_{S_{0}}}=u_{k}$ for some $s_{0} \in \Delta$ and some $k$.

Remark 5.4. The fact that this family can be contracted to a surface with normal points is proved in [22, Lemma 2.2.6].

Let $h$ be a defining holomorphic function of the divisor $H$ near the point of intersection $u(\Delta) \cap H$. Then $h \circ \mathcal{U}$ is holomorphic on $Y$ (for this one might need to take disks of smaller radii) and is equal to zero at $0 \in Y_{0} \subset Y$. At the same time it cannot vanish on $\bigcup_{s \in \Delta} \partial Y_{s}$ because $\left.\mathcal{U}\right|_{Y_{S}}\left(\partial Y_{s}\right)$ is close to $u(\partial \Delta)$ for all $s \in \Delta$. Therefore the zero set of $h \circ \mathcal{U}$ must intersect every $Y_{s}$. And that means that $u_{k}(\Delta)$ intersects $H$.

Let us make one more remark. Let $\omega_{F S}$ be the Fubini-Study form on $\mathbb{P}^{N}$. For a holomorphic map $f: \bar{\Delta} \rightarrow \mathbb{C}^{N}$ (we always suppose $f$ to be defined in a neighborhood of the closure $\bar{\Delta}$ ), the area of $f(\Delta)$ with respect to the Fubini-Study form is

$$
\operatorname{area}_{F S} f(\Delta)=\int_{\Delta} f^{*} \omega_{F S}
$$


Denote by $Z=\left(Z_{0}, \ldots, Z_{N}\right)$ coordinates in $\mathbb{C}^{N+1}$ and let $\pi: \mathbb{C}^{N+1} \backslash\{0\} \rightarrow \mathbb{P}^{N}$ be the standard projection. Consider the following singular $(1,1)$-form on $\mathbb{C}^{N+1}$

$$
\omega_{0}=d d^{c} \ln \|Z\|^{2} .
$$

The following statement is a simple case of King's residue formula, but we shall give a simple proof for the sake of completeness.

Lemma 5.5. For a holomorphic lift $F=\left(f^{0}, \ldots, f^{N}\right): \bar{\Delta} \rightarrow \mathbb{C}^{N+1}$ of $f: \bar{\Delta} \rightarrow$ $\mathbb{P}^{N}$ (i.e., $\left.f=\pi \circ F\right)$ such that $\left.F\right|_{\partial \Delta}$ does not vanish one has

$$
\operatorname{area}_{F S} f(\Delta)=\int_{\partial \Delta} d^{c} \ln \|F\|^{2}-N_{F} .
$$

Here $N_{F}$ is the number of zeroes of $F$ counted with multiplicities.

Proof. By the very definition of the Fubini-Study form one has $\pi^{*} \omega_{F S}=\omega_{0}$. And therefore it is immediate to check that in a neighborhood of a point $a \in \Delta$ such that $F(a) \neq 0$ one has that $f^{*} \omega_{F S}=F^{*} \omega_{0}$. As the result

$$
\operatorname{area}_{F S} f(\Delta)=\int_{\Delta} f^{*} \omega_{F S}=\int_{\Delta \backslash Z_{F}} F^{*} \omega_{0},
$$

where $Z_{F}:=\left\{z_{1}, \ldots, z_{k}\right\}$ is the set of zeroes of $F$, i.e., such $z_{l}$ that $f^{j}\left(z_{l}\right)=0$ for all $j=0, \ldots, N$. Let $n_{l}$ be the multiplicity of zero $z_{l}$. Then $F(z)=(z-$ $\left.z_{i}\right)^{n_{l}}\left(g^{0}(z), \ldots, g^{N}(z)\right)$, where at least one of $g^{j}$-s is not zero at $z_{l}$. We have that

$$
d d^{c} \ln \|F\|^{2}=n_{l} \delta_{z l}+d d^{c} \ln \|G\|^{2},
$$

where $G(z)=\left(g^{0}(z), \ldots, g^{N}(z)\right)$. Therefore $d d^{c} \ln \|G\|^{2}$ is an extension of $F^{*} \omega_{0}$ to $z_{l}$. The rest obviously follows from the Stokes formula.

Let us observe the following immediate corollary from this lemma.

Corollary 5.6. Let $f_{k}: U \rightarrow \mathbb{P}^{N}$ be a $\Gamma$-converging sequence of meromorphic mappings and let $L$ be a divisor in $U$ such that $f_{k}$ converge uniformly on compacts of $U \backslash L$. Let $V \cong \Delta^{n-1} \times \Delta$ be a scale adapted to $L$ and to the limit $M$ of $f_{k}^{*} H_{0}$, where $H_{0}=\left[Z_{0}=0\right]$. Then the areas of the analytic disks $f_{k}\left(\Delta_{z^{\prime}}\right)$ are uniformly bounded in $z^{\prime} \in \Delta^{n-1}$ and $k \in \mathbb{N}$.

Proof. Let $\left(z^{\prime}, z_{n}\right)$ be coordinates in $\Delta^{n-1} \times \Delta$. Denote by $F_{k}=\left(f_{k}^{0}, \ldots, f_{k}^{N}\right)$ lifts of $f_{k}$ to $\mathbb{C}^{N+1}$. Consider restrictions $\left.f_{k}\right|_{\Delta_{z^{\prime}}}$. Due to the fact that our chart is adapted to $M=\lim f_{k}^{*} H_{0}$ we have that $f_{k}^{0}$ does not vanish on $\partial \Delta_{z^{\prime}}$ for $k \gg 1$ and, since it is also adapted to $L$ the lifts $F_{k}=\left(f_{k}^{0}, \ldots, f_{k}^{N}\right)$ converge in a neighborhood of $\partial \Delta_{z^{\prime}}$. By (5.3) we have

$$
\operatorname{area}_{F S} f_{k}\left(\Delta_{z^{\prime}}\right) \leq \int_{\partial \Delta_{z^{\prime}}} d^{c} \ln \left\|F_{k}\right\|^{2} \leq c
$$

i.e., the areas are uniformly bounded for $z^{\prime} \in \Delta^{n-1}$ and all $k$. 
Remark 5.7. For a family $\mathcal{F}$ of meromorphic mappings from a manifold $U$ to a projective manifold $X$ to be normal an obvious necessary condition is that for any fixed hypersurface $H \subset X$ and any fixed compact $K \Subset U$ the volumes counting with multiplicities of intersections $f^{*} H \cap K$ should be uniformly bounded for $f \in$ $\mathcal{F}$. It was proved by Fujimoto in [10] that this condition (in the case $X=\mathbb{P}^{N}$ and $H_{i}$ are hyperplanes) turns out to be also sufficient, but only for the meromorphic (i.e., Г) normality. We in this paper are interested in the normality in the weak convergence sense (which is, that's to say, stronger than meromorphic one). In that case there is one more necessary condition. Take two hypersurfaces $H_{0}$ and $H_{1}$ in $X$. Let $\left\{f^{*} H_{0}: f \in \mathcal{F}\right\}$ and $\left\{f^{*} H_{1}: f \in \mathcal{F}\right\}$ be the families of their preimages by elements of our family $f \in \mathcal{F}$. By boundedness of volumes condition for every sequence $f_{k}^{*} H_{i}, i=0,1$, some subsequences $f_{k_{j}}^{*} H_{i}$ converge to divisors $L_{0}$ and $L_{1}$. If there exist coinciding (without taking to account the multiplicities) components $L_{0}^{\prime}$ and $L_{1}^{\prime}$ of $L_{0}$ and $L_{1}$ respectively, then $f_{k_{j}}$ cannot weakly converge in a neighborhood of $L_{0}^{\prime}=L_{1}^{\prime}$. Indeed, since the limit $f$ is a holomorphic map outside of $I_{f}$, the preimages $f^{*} H_{0}$ and $f^{*} H_{1}$ cannot have common components. But $L_{0}^{\prime}$ and $L_{1}^{\prime}$ are such components. Contradiction. This will be formalized in the following definition.

Let $\mathcal{F}$ be a $\Gamma$-normal family in $\mathcal{M}(U, X)$. Fix a divisor $H$ in $X$. Remark that for every relatively compact $D \Subset U$ the intersections $f^{*} H \cap \bar{D}$ from a pre-compact family of sets when $f$ is running over $\mathcal{F}$. Therefore one can find a finite collection of scales $\left\{E_{\alpha}\right\}$ such that every $f^{*} H \cap \bar{D}$ can be covered by some of corresponding $V_{\alpha}$ and the members of this covering are adapted to $f^{*} H$. This collection $\left\{E_{\alpha}\right\}$ of scales depends on $D \Subset U$, but does not depend on $f \in \mathcal{F}$ and, moreover, does not depend on $H$ taken in some compact family of divisors, in our case this family is $\left\{H_{0}, \ldots, H_{d}\right\}$, i.e., is finite.

Definition 5.8. We say that a family $\mathcal{F}$ of meromorphic mappings from a complex manifold $U$ to a complex manifold $X$ uniformly separates hypersurfaces $H_{0}$ and $H_{1}$ from $X$ if for any $f \in \mathcal{F}$ and any adapted for both $f^{*} H_{0}$ and $f^{*} H_{1}$ scale $E_{\alpha}=\left(V_{\alpha}, j_{\alpha}, \Delta^{n-1}, \Delta\right)$ as above, the Hausdorff distance between $f^{*} H_{0} \cap V_{\alpha}$ and $f^{*} H_{1} \cap V_{\alpha}$ for $f \in \mathcal{F}$ is bounded from below by a strictly positive constant.

Hausdorff distance is taken here in the Euclidean metric of $\mathbb{C}^{n}$. A constant in question may well depend on divisors $H_{0}, H_{1}$ and adapted chart $V_{\alpha}$, but it is supposed not to depend on $f \in \mathcal{F}$.

\subsection{Proof of the normality criterion of Theorem 1.8}

We are going to prove now Theorem 1.8 from the Introduction. Recall that a relatively compact open subset $Y$ of a complex manifold $X$ is said to be hyperbolically imbedded to $X$ if for any two sequences $\left\{x_{n}\right\}$ and $\left\{y_{n}\right\}$ in $Y$ converging to distinct points $x \in \bar{Y}$ and $y \in \bar{Y}$ one has

$$
\limsup _{n \rightarrow \infty} k_{Y}\left(x_{n}, y_{n}\right)>0,
$$


where $k_{Y}$ is the Kobayashi pseudodistance of $Y . \quad Y \Subset X$ is said to be locally hyperbolically complete (1.h.c) if for every $y \in \bar{Y}$ there exists a neighborhood $V_{y} \ni$ $y$ such that $V_{y} \cap Y$ is hyperbolically complete. For example every $Y \Subset X$ of the form $X \backslash\{$ divisor $\}$ is obviously 1.c.h. It was proved in [26] that if $Y$ is hyperbolically imbedded into $X$ and is 1.h.c. then $Y$ is complete hyperbolic.

These notions are connected to complex lines in $\bar{Y}$ by theorem of Zaidenberg, see [44]. By a complex line in $Y$ (or in $X$ ) one understands an image of a non-constant holomorphic map $u: \mathbb{C} \rightarrow Y$ (or $X$ ). Sometimes one requires that $\left\|d_{z} u\left(\frac{\partial}{x}\right)\right\|_{h} \leq 1$ for all $z \in \mathbb{C}$, where $h$ is some Hermitian metric on $X$. Complex line $u: \mathbb{C} \rightarrow \bar{Y} \Subset X$ is called limiting for $Y$ if there exists a sequence of holomorphic mappings $u_{n}: \Delta(R) \rightarrow Y$ converging on compacts in $\mathbb{C}$ to $u: \mathbb{C} \rightarrow \bar{Y}$. Theorem of Zaidenberg says now that: for a relatively compact 1.c.h. domain $Y$ in a complex manifold $X$ to be complete hyperbolic and hyperbolically imbedded in $X$ it is necessary and sufficient that $Y$ does not contain complex lines and does not admit limiting complex lines.

Now we turn to the proof. Let $\left\{f_{k}\right\}$ be a sequence from $\mathcal{F}$, where $\mathcal{F}$ satisfies the assumptions of Theorem 1.8 from the Introduction. $\left\{H_{i}\right\}_{i=0}^{d}$ is our set of divisors.

Step 1. Convergence outside of a divisor. By Bishop's compactness theorem for every $i$ some subsequence from $f_{k}^{*} H_{i}$ converges to a (may empty) hypersurface in $U$. Denote this limit hypersurface as $L_{i}$. Set

$$
L:=\bigcup_{i=0}^{d} L_{i}
$$

In order not to complicate notations we will not introduce subindexes when extracting subsequences.

If $L$ is empty then for every compact $K \Subset U$ all $f_{k}$ with $k$ big enough send $K$ to $X \backslash \bigcup_{i=0}^{d} H_{i}$, the last is Stein. In particular they are holomorphic in a neighborhood of $K$ and we can use Zalcman's Lemma 5.1 together with Zaidenberg's characterization to extract a converging subsequence.

Therefore from now on we suppose $L$ is nonempty. Take a point $z_{0} \in U \backslash L$ and take a relatively compact neighborhood $V \ni z_{0}$ biholomorphic to a ball such that $\bar{V} \cap L=\emptyset$. Then for $k$ big enough $f_{k}(\bar{V}) \subset X \backslash \bigcup_{i=0}^{d} H_{i}$. This implies that they all are holomorphic on $V$ and we again can find a converging subsequence on $V$ as before. Therefore some subsequence of $\left\{f_{k}\right\}$ (still denoted as $\left\{f_{k}\right\}$ ) converge on compacts of $U \backslash L$ in the usual sense of holomorphic mappings. Denote by $f$ its limit. $f$ is a holomorphic map from $U \backslash L$ to $X$.

Step 2. Convergence across the divisor. Take a point $z_{0} \in L_{0}$, if $L_{0}$ is empty we can re-numerate $L_{i}$-s. Fix an imbedding $i: X \rightarrow \mathbb{P}^{N}$ and let $H$ be the intersection of $X$ (i.e., of $i(X))$ with hyperplane $\left\{Z_{0}=0\right\}$ in the standard homogeneous coordinates $\left[Z_{0}: \ldots: Z_{N}\right]$ of $\mathbb{P}^{N}$. After going to a subsequence we have that $f_{k}^{*} H$ converge, denote by $M$ the limit. Let $(V, j)$ be an adapted chart for $L \cup M$ (and therefore also for $\left.L_{0}\right)$ at $z_{0}$ with the scale $E=\left(V, j, \Delta^{n-1}, \Delta\right)$. Let $P_{k}\left[z_{n}\right] \in \mathcal{O}_{\Delta^{n-1}}\left[z_{n}\right]$ be the 
defining unitary polynomial for $f_{k}^{*} H \cap V . P_{k}$ converges to the defining polynomial $P$ of $M \cap V$.

Let $f_{k}=\left[f_{k}^{0}: \ldots: f_{k}^{N}\right]$ be reduced representations of $f_{k}$ on $V$ (we write $f_{k}$ for $i \circ f_{k}$ ). Then multiplying this representation by the unit $P_{k} / f_{k}^{0}$ we obtain a reduced representation $f_{k}=\left[P_{k}: g_{k}^{1}: \ldots: g_{k}^{N}\right]$. We have that $g_{k}^{j} / P_{k}$ converge on compacts of $V \backslash(L \cup M)$. Therefore $g_{k}^{j}$ converge there to, denote by $g^{j}$ its limit. We see that lifts $F_{k}=\left(P_{k}, g_{k}^{1}, \ldots, g_{k}^{N}\right)$ converge to $F:=\left(P, g^{1}, \ldots, g^{N}\right)$ on compacts of $V \backslash(L \cup M)$. By maximum principle they converge on $V$.

In particular $f$ extends to a meromorphic mapping from $U$ to $X$.

Remark 5.9. It is worth noticing that at this stage we proved the $\Gamma$-normality of our family. For the case $X=\mathbb{P}^{N}$ with $H_{i}$ hyperplanes this was proved in [10]. One more point worth of noticing is that the extendibility of $f$ also follows from usual complex hyperbolic geometry, see [28].

Step 3. Convergence outside of codimension two. Changing indices of $H_{i}$, if necessary, we can suppose that our family uniformly separates $H_{0}$ and $H_{1}$. Take a point $z_{0} \in L_{0} \backslash \bigcup_{i \neq 0} L_{i}$ such that $L_{0}$ in addition is smooth at $z_{0}$. Take an adapted scale $E=\left(V, j, \Delta^{n-1}, \Delta\right)$ for $L_{0}$ near $z_{0}$ which intersects $L$ only by the smooth part of $L_{0}$ and, moreover, such that $j\left(L_{0} \cap V\right)=d \cdot\left[\Delta^{n-1} \times\{0\}\right]$ for some multiplicity $d \geq 1$. Fix coordinates $\left(z^{\prime}, z_{n}\right)$ on $\Delta^{n-1} \times \Delta$. By Corollary 5.6 the areas of analytic disks $\left.f_{k}\right|_{\Delta_{z^{\prime}}}$ are uniformly bounded. Fix some $z^{\prime} \in \Delta^{n-1}$ and take a subsequence $f_{k}$ such that $\left.f_{k}\right|_{\Delta_{z^{\prime}}}$ converge in stable topology to $\left.f\right|_{\Delta_{z^{\prime}}}$ plus a chain $C_{z^{\prime}}$ of rational curves. By Corollary 5.3 if $C_{z^{\prime}}$ intersects some $H_{1}$ with $i \neq 0$ then $\left.f_{k}\right|_{\Delta_{z^{\prime}}}\left(\Delta_{z^{\prime}}\right)$ intersects $H_{1}$ to. But then $f_{k}^{*} H_{1} \cap V$ is nonempty and converge to $L_{1} \cap V$. This can be only $L_{0} \cap V$ with some multiplicity, because $V$ was chosen in such a way that $L \cap V=L_{0} \cap V$. The last violates the assumed uniform separability of the pair $H_{0}, H_{1}$ by $\mathcal{F}$. Therefore $C_{z^{\prime}}$ is empty. That means that (some subsequence of) $\left.f_{k}\right|_{\Delta_{z^{\prime}}}$ uniformly on $\Delta_{z^{\prime}}$ converge to $\left.f\right|_{\Delta_{z^{\prime}}}$. This implies that the whole sequence $f_{k}$ restricted to $\Delta_{z^{\prime}}$ converge to $f$. Therefore $f_{k}$ converge to $f$ on $U \backslash \operatorname{Sing} L$ in compact open topology as holomorphic mappings. This proves the Theorem.

Remark 5.10. Theorem of Bloch, see also [13], states that $Y=\mathbb{P}^{N} \backslash \bigcup_{j=0}^{2 N} H_{i}$ is hyperbolically imbedded to $\mathbb{P}^{N}$, where $H_{i}$ are hyperplanes in general position. Therefore $Y=\mathbb{P}^{N} \backslash \bigcup_{i=0}^{2 N} H_{i}$ is an example for our Theorem 1.8.

\section{Behavior of volumes of graphs under weak and gamma convergence}

In this section we are concerned with the following question: let meromorphic mappings $f_{k}: U \rightarrow X$ converge in some sense to a meromorphic map $f$, what can be said about the behavior of volumes of graphs of $f_{k}$ over compacts in $U$ ? If $f_{k}$ converge to $f$ strongly then, as it was proved in Theorem 1.1, for every relative compact $V \Subset U$ we have that

$$
\operatorname{Vol}\left(\Gamma_{\left.f_{k}\right|_{V}}\right) \rightarrow \operatorname{Vol}\left(\Gamma_{\left.f\right|_{V}}\right) .
$$


When $f_{k}$ converges only weakly one cannot, of course expect anything like (6.1). At most what one can expect is that volumes of $\Gamma_{f_{k}}$ stay bounded over compacts in $U$ and converge to the volume of $\Gamma_{f}$ plus volumes of exceptional components. I.e., the question is if for a weakly converging sequence $\left\{f_{k}\right\}$ one has that for every relatively compact open $V \Subset U$ there exists a constant $C_{V}$ such that

$$
\operatorname{Vol}\left(\Gamma_{\left.f_{k}\right|_{V}}\right) \leq C_{V} \quad \text { for all } \quad k .
$$

This turns to be wrong in general, the following example was communicated to us by A. Rashkovskii.

\subsection{Example of Rashkovskii}

Example 6.1. There exists a sequence $\varepsilon_{k} \searrow 0$ such that holomorphic mappings $f_{k}: \mathbb{B}^{3} \rightarrow \mathbb{P}^{3}$ defined as

$$
f_{k}:\left(z_{1}, z_{2}, z_{3}\right) \rightarrow\left[z_{1}: z_{1}-\varepsilon_{k}: z_{2}: z_{3}^{k}\right]
$$

converge weakly to $f(z)=\left[z_{1}: z_{1}: z_{2}: 0\right]$ on compacts of the unit ball $\mathbb{B}^{3} \subset \mathbb{C}^{3}$, but the volumes of graphs of $f_{k}$ over the ball $\mathbb{B}^{3}(1 / 2)$ of radius $1 / 2$ diverge. In fact

$$
\operatorname{Vol}\left(\Gamma_{f_{k}}\right) \cap\left(\mathbb{B}^{3}(1 / 2) \times \mathbb{P}^{3}\right) \geq k
$$

Consider the following family of plurisubharmonic functions on the unit ball $\mathbb{B}^{3}$ in $\mathbb{C}^{3}$ :

$$
u_{\varepsilon, k}(z)=\ln \left(\left|z_{1}\right|^{2}+\left|z_{1}-\varepsilon\right|^{2}+\left|z_{2}\right|^{2}+\left|z_{3}\right|^{k}\right), \quad \varepsilon \in(0,1 / 4) .
$$

Note that every $u_{\varepsilon, k}$ is bounded in $\mathbb{B}^{3}$ and its total MA mass in $\mathbb{B}^{3}(1 / 2)$ coincides with those of the function

$$
\tilde{u}_{\varepsilon, k}:=\max \left\{u_{\varepsilon, k}, s_{k}\right\} \text { where } s_{k}=\min \left\{\ln \left(\left|z_{1}\right|^{2}+\left|z_{2}\right|^{2}+\left|z_{3}\right|^{k}\right): z \in \mathbb{S}^{5}(1 / 2)\right\} \text {. }
$$

Here $\mathbb{S}^{5}(1 / 2)=\partial \mathbb{B}^{3}(1 / 2)$ is the sphere of radius $1 / 2$. This fact follows from the Bedford-Taylor definition of the MA mass of a product of bounded psh functions, see [4]: $d d^{c} u_{1} \wedge d d^{c} u_{2}:=d d^{c}\left(u_{1} d d^{c} u_{2}\right)$ and so on by induction. Here the point is, of course, to prove that $d d^{c}\left(u_{1} d d^{c} u_{2}\right)$ is again a closed positive current. Now one writes

$$
\begin{aligned}
\operatorname{MA}_{\mathbb{B}^{3}(1 / 2)}\left(u_{\varepsilon, k}\right) & =\int_{\mathbb{B}^{3}(1 / 2)}\left(d d^{c} u_{\varepsilon, k}\right)^{3}=\int_{\mathbb{B}^{3}(1 / 2)} d d^{c} u_{\varepsilon, k} \wedge\left(d d^{c} u_{\varepsilon, k}\right)^{2} \\
& =\int_{\partial \mathbb{B}^{3}(1 / 2)} d^{c} u_{\varepsilon, k} \wedge\left(d d^{c} u_{\varepsilon, k}\right)^{2}=\int_{\partial \mathbb{B}^{3}(1 / 2)} d^{c} \tilde{u}_{\varepsilon, k} \wedge\left(d d^{c} \tilde{u}_{\varepsilon, k}\right)^{2} \\
& =\int_{\mathbb{B}^{3}(1 / 2)}\left(d d^{c} \tilde{u}_{\varepsilon, k}\right)^{3}=\operatorname{MA}_{\mathbb{B}^{3}(1 / 2)}\left(\tilde{u}_{\varepsilon, k}\right)
\end{aligned}
$$


because $u_{\varepsilon, k}=\tilde{u}_{\varepsilon, k}$ on the sphere $\mathbb{S}^{5}(1 / 2)$. Since $\tilde{u}_{\varepsilon, k}$ converge uniformly to $\tilde{u}_{k}=$ $\max \left\{\ln \left(2\left|z_{1}\right|^{2}+\left|z_{2}\right|^{2}+\left|z_{3}\right|^{k}\right), s_{k}\right\}$ as $\varepsilon \searrow 0$ and $\operatorname{MA}_{\mathbb{B}^{3}(1 / 2)}\left(\tilde{u}_{k}\right)=\operatorname{MA}_{\mathbb{B}^{3}(1 / 2)}\left(u_{k}\right)=$ $4 k$, where $u_{k}=\ln \left(2\left|z_{1}\right|^{2}+\left|z_{2}\right|^{2}+\left|z_{3}\right|^{k}\right)$, we shall have that for $\varepsilon_{k}$ small enough $\mathrm{MA}_{\mathbb{B}^{3}(1 / 2)}\left(u_{\varepsilon_{k}, k}\right) \geq k$. This finishes the proof.

Remark 6.2. Examples of psh functions with polar singularities and unbounded non-polar MA mass where constructed first by Shiffman and Taylor, see [39], and especially simple one by Kiselman, see [27]: $u\left(z_{1}, \ldots, z_{n}\right)=\left(1-\left|z_{n}\right|^{2}\right)\left(-\ln \left\|z^{\prime}\right\|^{2}\right)^{1 / 2}$ for $z^{\prime}=\left(z_{1}, \ldots, z_{n-1}\right)$. Taking any of these examples and smoothing it by convolutions one gets a decreasing sequences of psh functions converging outside of an analytic set (on any codimension) to a psh function, smooth outside of this set with unbounded non-polar MA mass. The remarkable feature of the example of Rashkovskii, just described, is that functions in this example have a geometric meaning, their $d d^{c}$-s are pullbacks of Fubini-Study form by a meromorphic mappings to the complex projective space, i.e., the sum of their non-polar MA masses are the volumes of the corresponding graphs.

\subsection{Case of dimensions one and two}

If $\left\{f_{k}\right\}$ is a $\Gamma$-converging sequence of meromorphic mappings with values in one dimensional complex manifold then it is easy to see that the volumes of graphs of $f_{k}$-s are locally bounded over compacts in the source. Indeed, a one dimensional manifold $X$ either properly imbeds to $\mathbb{C}^{n}$ (when $X$ is noncompact) or is projective and therefore imbeds to $\mathbb{P}^{n}$. In both cases by Theorem 1.3 we have convergence of reduced representations to a, may be nonreduced representation of the limit. Inequality (5.5) implies that in an appropriately chosen local coordinates $\left(z^{\prime}, z_{n}\right)$ one has

$$
\begin{aligned}
\operatorname{Vol}\left(\Gamma_{\left.f_{k}\right|_{\Delta^{n}}}\right) & =\int_{\Delta^{n}}\left(d d^{c}\|z\|^{2}\right)^{n}+\int_{\Delta^{n}}\left(d d^{c}\|z\|^{2}\right)^{n-1} \wedge f_{k}^{*} \omega_{F S} \\
& \leq \int_{\Delta^{n}}\left(d d^{c}\|z\|^{2}\right)^{n}+\int_{\Delta^{n-1}}\left(d d^{c}\|z\|^{2}\right)^{n-1} \int_{\partial \Delta_{z^{\prime}}} d^{c} \ln \left\|F_{k}\right\|^{2} \leq \text { const. }
\end{aligned}
$$

Next, if the dimension $n$ of the source $U$ is 2 the boundedness of volumes of graphs of a weakly converging sequence is automatic. This can be seen at least in two ways. First, in projective case this readily follows from the following formula of King, see [25]:

$d\left[d^{c} \ln \left(\|f\|^{2}\right) \wedge d d^{c} \ln \left(\|f\|^{2}\right)\right]=\chi_{U \backslash I_{f}}\left[\left(d d^{c} \ln \left(\|f\|^{2}\right)\right)^{2}\right]-\sum_{j} n_{j}\left[Z_{j}\right]$,

provided $I_{f}$ has pure codimension two. $Z_{j}$ are irreducible components (branches) of the indeterminacy set $I_{f}$ of $f$. If it has branches of higher codimension then around these branches a higher order non-pluripolar masses can be expressed in a similar way. Now if $f_{k}$ weakly converge to $f$ formula (6.6) immediately gives a 
uniform bound of corresponding MA masses (even together with that concentrated on pluripolar sets $I_{f_{k}}$ ). If $n=2$ then that's all we need.

Second, using Skoda potentials, or Green functions, as it was done in [18, Theorem 2], one can bound non-pluripolar Monge-Ampère masses of order two also in the case of weakly converging sequence with values in disk-convex Kähler $X$. This observation implies that if $X$ is disk-convex Kähler and $\operatorname{dim} U=2$ then the volumes of graphs of weakly converging sequences of meromorphic mappings $U \rightarrow X$ are uniformly bounded over compacts in $U$.

Moreover, it was proved in [32] that volumes of weakly converging sequence are bounded also in the case when $X$ is any compact complex surface. The proof uses Kaähler case separately and then the fact that a non-Kähler surface has only finitely many rational curves.

Remark 6.3. Let us remark that there is one more important case when the volumes of graphs of weakly (even $\Gamma$ ) converging sequence necessarily stay bounded: namely when $\left\{f_{k}\right\}$ is a $\Gamma$-converging sequence of meromorphic mappings between projective manifolds $X$ and $Y$. Indeed the volumes of graphs $\Gamma_{f_{k}}$ are uniformly bounded as it is straightforward from Besout theorem.

\section{Rational connectivity of the exceptional components of the limit}

\subsection{Chains of rational curves}

Recall that a rational curve $C$ in a complex manifold $X$ is an image of $\mathbb{P}^{1}$ in $X$ under a non-constant holomorphic map $h: \mathbb{P}^{1} \rightarrow X$. A chain of rational curves is a connected union $C=\bigcup_{j} C_{j}$ of finitely many rational curves.

Definition 7.1. A closed subset $\Gamma \subset X$ we call rationally connected if for very two points $p \neq q$ in $\Gamma$ there exists a chain of rational curves $C \subset \Gamma$ such that $p, q \in C$.

One says also that $C$ connects $p$ with $q$. If $\Gamma$ is a complex manifold then this property is equivalent to the either of the following two ones:

- Every two points in $X$ can be connected by a single rational curve.

- For any finite set of points $F \subset X$ there exists a rational curve $C \supset F$.

We refer to [1] for these facts. Now let us turn to the proof of Theorem 1.9 from the Introduction. It consists from the two following lemmas. Let $f_{k}$ be a weakly or, gamma-converging sequence of meromorphic mappings and $f$ denotes their limit. Let $\hat{\Gamma}$ be the Hausdorff limit of the graphs, $\Gamma=\overline{\hat{\Gamma} \backslash \Gamma_{f}}$ the corresponding bubble. Set $\gamma:=\operatorname{pr}_{1}(\Gamma)$. It is at most a divisor in $\Gamma$-case and has codimension $\geq 2$ in the weak case. Let $V \cong \Delta^{n-1} \times \Delta$ be a scale adapted to $\gamma$ in the sense that $\left(\bar{\Delta}^{n-1} \times \Delta\right) \cap \gamma=\emptyset$.

Lemma 7.2. Suppose that there exists a dense subset $S \subset \Delta^{n-1}$ such that the areas of the analytic disks $\Gamma_{\left.f_{k}\right|_{z_{z^{\prime}}}}$ are uniformly bounded in $z^{\prime} \in S$ and $k \in \mathbb{N}$, then for every point $a \in \gamma$ the fiber $\Gamma_{a}:=\operatorname{pr}_{2}\left(\operatorname{pr}_{1}^{-1}(a)\right)$ is rationally connected. 
Proof. Here writing $\left.f_{k}\right|_{\Delta_{z^{\prime}}}$ we mean the restriction of $f_{k}$ to the disk $\Delta_{z^{\prime}}:=\left\{z^{\prime}\right\} \times \Delta$. Fix a point $a \in \gamma$ and some $a^{1}, a^{2} \in \Gamma_{a}$. Suppose $a^{1} \neq a^{2}$, otherwise there is nothing to prove. We need to prove that there exists a chain of rational curves in $\Gamma_{a}$ connecting $a^{1}$ with $a^{2}$. Since $\Gamma_{f_{k}}$ converge to $\hat{\Gamma} \supset \Gamma_{a}$ there exist $a_{k}^{1} \rightarrow a$ and $a_{k}^{2} \rightarrow a$ such that $f_{k}\left(a_{k}^{1}\right) \rightarrow a^{1}$ and $f_{k}\left(a_{k}^{2}\right) \rightarrow a^{2}$. Perturbing slightly we can take such $a_{k}^{i}$ to be regular (i.e., not indeterminacy) points of $f_{k}$ for $i=1,2$. Take a scale adapted to $\gamma$ near $a$ in the sense that $\gamma \cap\left(\Delta^{n-1} \times \partial \Delta\right)=\emptyset$. Denote by $\left(z^{\prime}, z_{n}\right)=\left(z_{1}, \ldots, z_{n-1}, z_{n}\right)$ the corresponding coordinates and assume without loss of generality that $a=0$.

Let $b_{k}^{1} \rightarrow 0^{\prime}$ and $b_{k}^{2} \rightarrow 0^{\prime}$ in $\Delta^{n-1}$ be such that $a_{k}^{1} \in \Delta_{b_{k}^{1}}$ and $a_{k}^{2} \in \Delta_{b_{k}^{2}}$. Taking again $a_{k}^{i}$ sufficiently general we can arrange that $b_{k}^{i} \in S$ and disks $\Delta_{b_{k}^{1}}$ and $\Delta_{b_{k}^{2}}$ converge to the disk $\Delta_{0^{\prime}}$. After taking a subsequence we get that graphs in question converge to the graph $\Gamma_{\left.f\right|_{\Delta_{0^{\prime}}}} \cup C^{i}$, where $C^{i} \subset\{a\} \times X$ are chains of rational curves. Both these chains contain the point $\left.f\right|_{\Delta_{0^{\prime}}}(a)$. Therefore $C:=C^{1} \cup C^{2}$ is connected. At the same time by construction $C^{i} \ni a^{i}$.

\subsection{Proof of Theorem 1.9}

Let us first consider the case of $\Gamma$-converging sequence of meromorphic mappings with values in projective $X$. Corollary 5.6 gives us the required boundedness of ares of analytic disks which makes possible to apply Lemma 7.2 just proved. This gives us the statement of Theorem 1.9 for $\Gamma$-converging sequences of meromorphic mappings with values in projective manifolds.

To treat the case of Gauduchon target manifolds we shall need one more lemma.

Lemma 7.3. Let $\mathcal{F}$ be a family of meromorphic mappings from $\Delta^{n}$ to a disk-convex manifold $X$, which admits a pluriclosed metric form. Suppose that for some $0<$ $\epsilon<1$, the family $\mathcal{F}$ is holomorphic and equicontinuous on the Hartogs figure $H_{\varepsilon}^{n}$. Then for every $0<r<1$ the areas of graphs $\Gamma_{f_{z^{\prime}}}$ of restrictions $f_{z^{\prime}}:=\left.f\right|_{\Delta_{z^{\prime}}(r)}$ of $f \in \mathcal{F}$ to the disks $\Delta_{z^{\prime}}(r):=\left\{z^{\prime}\right\} \times \Delta_{r}$ are uniformly bounded in $z^{\prime} \in \Delta_{r}^{n-1}$ and $f \in \mathcal{F}$.

Proof. For $f: \Delta^{n} \longrightarrow X$ a meromorphic map, we denote by $I_{f} \subset \Delta^{n}$ the set of points of indeterminacy of $f$. Since we suppose that all $f \in \mathcal{F}$ are holomorphic on $H_{\varepsilon}^{n}$ the sets $I_{f}$ do not intersect $\Delta^{n-1} \times A_{1-\varepsilon, 1}$. Consider currents $T_{f}=f^{*} \omega$ on $\Delta^{n}$, where $\omega$ is a pluriclosed metric form on $X$. Write

$$
T_{f}=\frac{i}{2} t_{f}^{\alpha \bar{\beta}} d z_{\alpha} \wedge d \overline{z_{\beta}}
$$

where $t_{f}^{\alpha \bar{\beta}}$ are distributions on $\Delta^{n}$ (in fact measures), smooth on $\Delta^{n} \backslash I_{f} \supset H_{\varepsilon}^{n}$. Fix $1-\varepsilon<r<r_{1}<1$ and consider on $\Delta^{n-1} \backslash \pi\left(I_{f}\right)$ (where $\pi: \Delta^{n} \rightarrow \Delta^{n-1}$ is 
the canonical projection onto the first factor) the area functions $a_{f}$ given by

$$
a_{f}\left(z^{\prime}\right)=\operatorname{area} f_{z^{\prime}}\left(\Delta_{r_{1}}\right)=\int_{\Delta_{z^{\prime}}\left(r_{1}\right)} T_{f}=\frac{i}{2} \int_{\Delta_{z^{\prime}}\left(r_{1}\right)} t_{f}^{n \bar{n}} d z_{n} \wedge d \overline{z_{n}} .
$$

Functions $a_{f}$ are well-defined and smooth on $\Delta^{n-1} \backslash \pi\left(I_{f}\right)$.

The proof of proposition will be done in two steps.

Step 1. Distributions $t_{f}^{n \bar{n}}$ are locally integrable in $\Delta^{n}$. Note that forms $T_{f}$ are smooth on $H_{\varepsilon}^{n}$ and the family $\left\{T_{f}: f \in \mathcal{F}\right\}$ is equicontinuous there. The condition that $d d^{c} T_{f}=0$ implies, in particular, that for all $1 \leq k, l \leq n-1$ one has

$$
\frac{\partial^{2} t_{f}^{n \bar{n}}}{\partial z_{k} \partial \bar{z}_{l}}+\frac{\partial^{2} t_{f}^{k \bar{l}}}{\partial z_{n} \partial \bar{z}_{n}}-\frac{\partial^{2} t_{f}^{k \bar{n}}}{\partial z_{n} \partial \overline{z_{l}}}-\frac{\partial^{2} t_{f}^{n \bar{l}}}{\partial z_{k} \partial \overline{z_{n}}}=0 .
$$

From (7.2) we get that on $\Delta^{n-1} \backslash \pi\left(I_{f}\right)$ :

$$
\begin{aligned}
& d d^{c} a_{f}=\left(\frac{i}{2}\right)^{2} \sum_{k, l=1}^{n-1}\left(\int_{\Delta_{z^{\prime}}\left(r_{1}\right)} \frac{\partial^{2} t_{f}^{n \bar{n}}}{\partial z_{k} \partial \bar{z}_{l}} d z_{n} \wedge d \overline{z_{n}}\right) d z_{k} \wedge d \bar{z}_{l} \\
& =\left(\frac{i}{2}\right)^{2} \sum_{k, l=1}^{n-1} \int_{\Delta_{z^{\prime}}\left(r_{1}\right)}\left(\frac{\partial^{2} t_{f}^{k \bar{n}}}{\partial z_{n} \partial \overline{z_{l}}}+\frac{\partial^{2} t_{f}^{n \bar{l}}}{\partial z_{k} \partial \overline{z_{n}}}-\frac{\partial^{2} t_{f}^{k \bar{l}}}{\partial z_{n} \partial \overline{z_{n}}}\right) d z_{n} \wedge d \overline{z_{n}} \cdot d z_{k} \wedge d \bar{z}_{l} \\
& =\left(\frac{i}{2}\right)^{2} \sum_{k, l=1}^{n-1}\left(\int_{\partial \Delta_{z^{\prime}\left(r_{1}\right)}} \frac{\partial t_{f}^{k \bar{n}}}{\partial \overline{z_{k}}} d \overline{z_{n}}+\int_{\partial \Delta_{z^{\prime}}\left(r_{1}\right)} \frac{\partial t_{f}^{n \bar{l}}}{\partial z_{k}} d z_{n}-\int_{\partial \Delta_{z^{\prime}}\left(r_{1}\right)} \frac{\partial t_{f}^{k \bar{l}}}{\partial \overline{z_{n}}} d \overline{z_{n}}\right) d z_{k} \wedge d \bar{z}_{l} \\
& =: \varphi_{f} .
\end{aligned}
$$

Forms $\varphi_{f}$ are smooth in the whole unit polydisk $\Delta^{n-1}$ and equicontinuous there because forms $T_{f}$ are smooth in $\Delta^{n-1} \times A_{1-\varepsilon, 1} \subset H_{\varepsilon}^{n}$ and equicontinuous there. Let us find a smooth and equicontinuous family on $\Delta_{r}^{n}$ of solutions $\psi_{f}$ of

$$
d d^{c} \psi_{f}=\varphi_{f}
$$

Set

$$
h_{f}:=a_{f}-\psi_{f} .
$$

Since $a_{f}$ is positive on $\Delta^{n-1} \backslash \pi\left(I_{f}\right)$ and $\psi_{f}$ is smooth on $\Delta^{n-1}$ we see that $h_{f}$ is bounded on $\Delta^{n-1}$ from below. Also $d d^{c} h_{f}=0$ on $\Delta^{n-1} \backslash \pi\left(I_{f}\right)$ and therefore $h_{f}$ extends to a plurisuperharmonic function on $\Delta^{n-1}$. This implies that $h_{f} \in$ $L_{l o c}^{1}\left(\Delta^{n-1}\right)$ see [16]. It follows that $a_{f}$ and $t_{f}^{n \bar{n}}$ are locally integrable. Step 1 is proved.

Step 2. Under the hypotheses of Lemma 7.3 functions $a_{f}$ defined by (7.1) are smooth on $\Delta^{n-1}$ and for every fixed $r<1$ the family $\left\{a_{f}\right\}_{f \in \mathcal{F}}$ is equicontinuous on $\bar{\Delta}_{r}^{n-1}$. 
Function $h_{f}$ given by (7.5) is plurisuperharmonic in $\Delta^{n-1}$ and pluriharmonic on $\Delta^{n-1} \backslash \pi\left(I_{f}\right)$. Therefore by Siu's lower semicontinuity of the level sets of Lelong numbers we have

$$
d d^{c} h_{f}=-\sum_{A \text { irr.comp. of } \pi\left(I_{f}\right)} c_{A}(f)[A],
$$

where $c_{A}(f) \geq 0$ and $[A]$ denotes the current of integration over the irreducible component $A$ of $\pi\left(I_{f}\right)$ of codimension one.

Remark 7.4. Note that through components of higher codimension a pluriharmonic function $h_{f}$ extends (as a pluriharmonic function). Therefore in (7.6) the sum is taken over the components of codimension one only.

We need to prove that $c_{A}(f)=0$. From (7.5) we get

$$
d d^{c} a_{f}=d d^{c} \psi_{f}-\sum_{A \text { irr.comp. of } \pi\left(I_{f}\right)} c_{A}(f)[A],
$$

where $d d^{c}$ from $a_{f}$ is taken in the sense of distributions (as from $L_{l o c}^{1}$-function). Let $\left\{h_{A}\right\}$ be equations of $A$. By Poincaré formula, see [12], [A] $=d d^{c} \ln \left|h_{A}\right|^{2}$ and therefore (7.7) writes as

$$
d d^{c} a_{f}=d d^{c} \psi_{f}-\sum_{A \text { irr.comp. of } \pi\left(I_{f}\right)} c_{A}(f) d d^{c} \ln \left|h_{A}\right|^{2}
$$

Take an one dimensional disk $\Delta$ in $\Delta^{n-1}$ which intersects $\pi\left(I_{f}\right)$ transversely at points $\left\{z_{j}\right\}$. Then (7.7) gives for restrictions of $a_{f}$ and $\phi_{f}$ to $\Delta$ (and we shall denote them by the same letters) the following

$$
\Delta a_{f}=\Delta \psi_{f}-\sum_{z_{j} \in \pi\left(A_{f}\right)} c_{j}(f) \delta_{z_{j}(f)} .
$$

Fix $\delta>0$ such that $\Delta\left(\delta, z_{j}\right)$ are pairwise disjoint. Let $\phi$ be a test function on $\Delta$ with support in $\Delta\left(\delta, z_{j}\right)$ for some fixed $j$. The coordinate on $\Delta$ denote as $z_{1}$.

Set

$$
a_{f}^{\epsilon}\left(z_{1}\right)=\frac{i}{2} \int_{\Delta_{z_{1}}\left(r_{1}\right)} t_{f, \epsilon}^{n \bar{n}} d z_{n} \wedge d \overline{z_{n}},
$$

where $t_{f, \epsilon}^{n \bar{n}}$ is the smoothing of $t_{f}^{n \bar{n}}$ by convolution. Since $t_{f, \epsilon}^{n \bar{n}} \rightarrow t_{f}^{n \bar{n}}$ in $L_{l o c}^{1}$ we get by Fubini Theorem that $a_{f}^{\epsilon} \rightarrow a_{f}$ in $L_{l o c}^{1}$. Therefore using (7.3) for dimension two 
we obtain

$$
\begin{aligned}
\left\langle\Delta a_{f}^{\epsilon}, \phi\right\rangle= & \frac{i}{2} \int_{\Delta\left(\delta, z_{j}\right)} \phi\left(z_{1}\right)\left(\int_{\Delta_{z_{1}}\left(r_{1}\right)} \frac{\partial^{2} t_{f, \epsilon}^{n \bar{n}}}{\partial z_{1} \partial \overline{z_{1}}} d z_{n} \wedge d \overline{z_{n}}\right) d z_{1} \wedge d \bar{z}_{1} \\
= & \frac{i}{2} \int_{\Delta\left(\delta, z_{j}\right)} \phi\left(z_{1}\right)\left(\frac{i}{2} \int_{\partial \Delta_{z_{1}}\left(r_{1}\right)} \frac{\partial t_{f, \epsilon}^{1 \overline{2}}}{\partial \overline{z_{1}}} d \overline{z_{2}}+\frac{i}{2} \int_{\partial \Delta_{z_{1}}\left(r_{1}\right)} \frac{\partial t_{f, \epsilon}^{2 \overline{1}}}{\partial z_{1}} d z_{2}\right) d z_{1} \wedge d \bar{z}_{1} \\
& -\frac{i}{2} \int_{\Delta\left(\delta, z_{j}\right)} \phi\left(z_{1}\right)\left(\int_{\partial \Delta_{z_{1}}\left(r_{1}\right)} \frac{\partial \bar{t}_{f, \epsilon}^{1 \overline{1}}}{\partial \overline{z_{2}}} d \overline{z_{2}}\right) d z_{1} \wedge d \bar{z}_{1} \longrightarrow<\varphi_{f}, \phi>
\end{aligned}
$$

as $\epsilon \longrightarrow 0$. Therefore, $\Delta a_{f}=\varphi_{f}$ in $\Delta$ in the sense of distributions. By regularity of the Laplacian $a_{f} \in \mathcal{C}^{\infty}$ on $\Delta$ and therefore $c_{A}(f)=0$ for all $A$ and all $f$. Therefore $a_{f}$ are smooth on $\Delta^{n-1}$ and $a_{f}=\psi_{f}+h_{f}$ there. $\psi_{f}$-s are equicontinuous and $h_{f}$ are pluriharmonic everywhere and uniformly bounded from below. Moreover $a_{f}$ are equicontinuous on $\Delta_{\varepsilon}^{n-1}$ by assumption. Therefore $h_{f}$ are equicontinuous on $\Delta_{\varepsilon}^{n-1}$. This implies equicontinuity of $h_{f}$ on compacts of $\Delta^{n-1}$, and therefore the equicontinuity of $a_{f}$. Step 2 and therefore our Lemma are proved.

Lemmas 7.2 and 7.3 obviously imply the Theorem 1.9 from the Introduction for the case of weakly converging sequences of meromorphic mappings with values in disk-convex Gauduchon manifolds.

\section{Fatou components}

\subsection{Case of dimension two and Fatou sets}

First let us prove two lemmas.

Lemma 8.1. Suppose that a weakly converging sequence $\left\{f_{k}\right\}$ of meromorphic mappings from a two-dimensional domain $U$ to a compact complex surface $X$ does not converge strongly. Then $X$ is bimeromorphic to $\mathbb{P}^{2}$.

Proof. Indeed, in that case there exists a point $a \in U$ and a neighborhood $V \ni a$ such that $f_{k}$ converge uniformly on compacts of $V \backslash\{a\}$ but $\Gamma_{f_{k}}$ do not converge to $\Gamma_{f}$, where $f: U \rightarrow X$ is the limit map. $\operatorname{Vol}\left(\Gamma_{f_{k}}\right)$ are uniformly bounded. Indeed, for Kähler $X$ it was proved in [18] using Skoda's potentials. In [32] its was proved for non-Kähler $X$ using that fact that such $X$ can contain only finitely many rational curves as well as existence of certain $d d^{c}$-exact $(2,2)$-forms.

Therefore we see that the limit $\hat{\Gamma}=\lim \Gamma_{f_{k}}$ contains $\Gamma_{f}$ plus $\{a\} \times X$ (with some multiplicity). But this is a bubble and therefore $X$ is rationally connected by Theorem 1.9. From the classification of surfaces, see [3], we know that such $X$ must be bimeromorphic to $\mathbb{P}^{2}$. 
For a meromorphic map $f: U \rightarrow X$ denote by

$$
D_{f}:=\operatorname{pr}_{1}\left(\left\{(z, x) \in U \times X: \operatorname{dim}_{(z, x)} \operatorname{pr}_{2}^{-1}(x) \geq 1\right\}\right)
$$

the set of degeneration of $f . f: U \rightarrow X$ is degenerate if $D_{f}=U$.

Lemma 8.2. Let $f: X \rightarrow X$ be a non-degenerate meromorphic self-map of a compact complex surface $X$ and let $z \in \Phi_{s}$ (respectively $\left.\Phi_{w}\right)$. Then for every $l \geq 1$ one has

$$
\left.\left.f^{l}[z] \backslash f^{l}\right|_{f^{l}}\left(D_{f^{l}}\right) \subset \Phi_{s} \quad \text { (respectively } \Phi_{w}\right) .
$$

Proof. Take some $\left.a \in f^{l}[z] \backslash f^{l}\right|_{D_{f^{l}}}\left(D_{f^{l}}\right)$. Since $f^{l}$ does not contract any curve to $a$ there exist neighborhoods $V \ni z$ and $U \ni a$ such that $\mathrm{pr}_{2}:(V \times U) \cap \Gamma_{f^{l}} \rightarrow U$ is proper. That means that $\left(f^{l}\right)^{-1}: U \rightarrow V$ is well defined as a multivalued holomorphic map. Now let $\left\{f^{n_{k}}\right\} \subset\left\{f^{n}\right\}$ be a subsequence. By assumption from the sequence $\left\{f^{n_{k}+l}\right\}$ we can subtract a strongly/weakly converging on $V$ subsequence $\left\{f^{n_{k_{j}}+l}\right\}$. That means that $\left\{f^{n_{k_{j}}}=f^{n_{k_{j}}+l} \circ f^{-l}\right\}$ will converge in an appropriate sense on $U$.

Let us turn to the proof of Corollary 1.10 from the Introduction. Since every compact complex surface admits a $d d^{c}$-closed metric form Theorem 1.5 applies in our case and gives local pseudoconvexity of the weak Fatou set $\Phi_{w}$. Suppose now that $\Phi_{s} \neq \Phi_{w}$.

a) By Lemma $8.1 X \simeq \mathbb{P}^{2}$.

b) There exist a point $p \in X$, a ball $B$ centered at $p$, a subsequence of iterates $\left\{f^{n_{k}}\right\}$, which uniformly converges on compacts of $\bar{B} \backslash\{p\}$ to a meromorphic map $f_{\infty}: \bar{B} \rightarrow X$, holomorphic on $B \backslash\{p\}$, but not converges strongly on any neighborhood of $p$. In particular this means that $p \in I\left(f_{\infty}\right)$ by Rouché Principle of [18] and, moreover, $C=f_{\infty}[p]$ is a chain of rational curves $\bigcup_{i=1}^{N} C_{i}$. As it was said $\operatorname{Vol}\left(\Gamma_{f^{n}}\right)$ are uniformly bounded on $\bar{B}$. So $\Gamma_{f^{n_{k}}}$ converge (after going to a subsequence) in cycle topology to $\Gamma_{f_{\infty}} \cup d(\{p\} \times X)$ for some integer $d \geq 1$. In particular $f$ cannot be degenerated in this case. Take a point $q \in X \backslash C$. Then for $k \gg 1$ we have that $q \in f^{n_{k}}(B \backslash\{p\})$. If moreover $q \notin f^{n_{k}}\left(D\left(f^{n_{k}}\right)\right)$ then $q \in \Phi_{w}$. But $\bigcup_{k} f^{n_{k}}\left(D\left(f^{n_{k}}\right)\right)$ is at most countable set of points and $\Phi_{w}$ is Levi-pseudoconvex. So $\Phi_{w} \supset X \backslash C$. Again from pseudoconvexity of $\Phi_{w}$ it follows that if $\Phi_{w}$ intersects some irreducible component of $C$ then it contains this component minus the rest of C. I.e., $\Phi_{w}=\mathbb{P}^{2} \backslash\{$ some components of $C\}$.

c) Take a point $(p, x) \in\{p\} \times X$ such that $x \in C$. Suppose that $\Gamma_{f_{\infty}} \cap(X \times\{x\})$ has $(p, x)$ as isolated point. Then we can find neighborhoods $W \ni p$ and $V \ni x$ such that $(\partial W \times \bar{V}) \cap \Gamma_{f_{\infty}}=\emptyset$. Therefore $(\partial W \times \bar{V}) \cap \Gamma_{f^{n} k}=\emptyset$ for $k$ big enough This means that $\Phi_{w} \supset V$ as before and, moreover, $\Phi_{w}$ contains the component of $C$ passing through $x$ minus the rest of $C$.

To finish the proof let us distinguish two cases. 
Case 1. Every component of $C$ contains such a point. In this case our sequence $\left\{f^{n_{k}}\right\}$ strongly converges on $X \backslash\{$ finite set $\}$. Furthermore, $\operatorname{Vol}\left(\Gamma_{f^{n}}\right)$ are uniformly bounded. Since they can't be less than $(\operatorname{deg} f)^{n_{k}} \cdot \operatorname{Vol}(X)$, we see that $f$ has degree one, and $f_{\infty}$ is a degenerate map to $C$ because $\Gamma_{f_{\infty}}$ has zero volume in this case. Moreover $f_{\infty}$ cannot be holomorphic near $p$, otherwise $f^{n_{k}}$ would converge strongly in a neighborhood of $p . C$ in this case should consist only from one component as a meromorphic image of an irreducible variety.

Case 2. There exists a component $C_{1}$ of $C$ such that for all points $x \in C_{1} \backslash \cup_{i \neq 1} C_{i}$ $\operatorname{dim}_{(p, x)} \Gamma_{f_{\infty}} \cap(X \times\{x\})>0$. Then $f_{\infty}$ is a degenerate mapping of $X$ onto this $C_{1}$ and therefore again $C_{1}$ is a single component of $C$. Indeed, any other component $C_{2}$ of $C$ should contain a point $x$ as above, because the image of $f_{\infty}$ should be irreducible. I.e., in both cases $C$ consists from one rational curve only.

The following simple example shows that the situation described in part (b) of this Corollary can really happen. Let $X=\mathbb{P}^{2}$ and $f:\left[z_{0}: z_{1}: z_{2}\right] \rightarrow\left[z_{0}: 2 z_{1}:\right.$ $\left.2 z_{2}\right]$. Then for this $f$ we have the phenomena described above with $p=[1: 0: 0]$ and $C=\left\{z_{0}=0\right\}$.

\subsection{Example}

Let us give one more example relevant to the Fatou sets.

Example 8.3. Consider the following rational self-map of $\mathbb{P}^{2}$ :

$$
f:\left[z_{0}: z_{1}: z_{2}\right] \rightarrow\left[z_{0}^{2} z_{1}: z_{1}^{3}: z_{0}^{2} z_{2}\right] .
$$

By induction one easily checks that

$$
f^{k}:\left[z_{0}: z_{1}: z_{2}\right] \rightarrow\left[z_{0}^{2^{k}} z_{1}^{2^{k}-1}: z_{1}^{2^{k+1}-1}: z_{0}^{2^{k+1}-2} z_{2}\right] .
$$

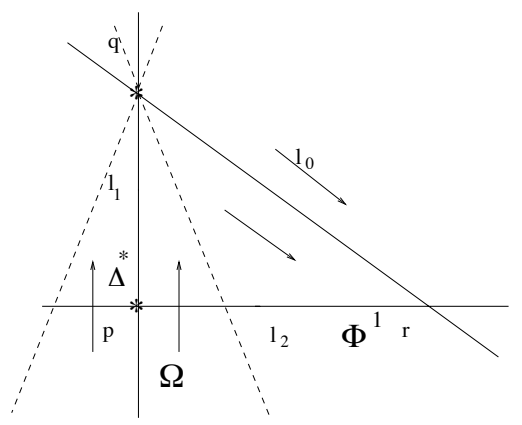

Figure 8.1. Mapping $f$ contracts the line $l_{1}:=\left\{z_{1}=0\right\}$ to the first of its points of indeterminacy $q=[0: 0: 1]$, line at infinity $l_{0}:=\left\{z_{0}=0\right\}$ to the regular point $r=[0: 1: 0]$ and do not contract anything to its second point of indeterminacy $p=[1: 0: 0]$. Levi flat cone $=$ Julia set for $f$ is marked by two punctured lines. 
Cover $\mathbb{P}^{2}$ by three standard affine charts $U_{i}=\left\{z_{i} \neq 0\right\}$ with coordinates $u_{1}=$ $\frac{z_{1}}{z_{0}}, u_{2}=\frac{z_{2}}{z_{0}}, v_{1}=z_{0} / z_{1}, v_{2}=z_{2} / z_{1}$ and $w_{1}=z_{0} / z_{2}, w_{2}=z_{1} / z_{2}$ respectively. Mapping $f: U_{0} \rightarrow U_{0}$ writes as

$$
f:\left(u_{1}, u_{2}\right) \rightarrow\left(u_{1}^{2}, u_{2} / u_{1}\right)
$$

We see from here that $f$ has degree 2. Furthermore $f^{k}$ writes as

$$
f^{k}:\left(u_{1}, u_{2}\right) \rightarrow\left(u_{1}^{2^{k}}, u_{2} / u_{1}^{2^{k}-1}\right)
$$

In the charts $f: U_{1} \rightarrow U_{1}$ our iterate writes as

$$
f^{k}:\left(v_{1}, v_{2}\right) \rightarrow\left(v_{1}^{2^{k}}, v_{1}^{2^{k+1}-2} v_{2}\right) \rightarrow(0,0)=r \quad \text { on } \quad\left\{\left|v_{1}\right|<1\right\}
$$

I.e., we see that $\Phi^{1}=\left\{\left|v_{1}\right|<1\right\}=\left\{\left|u_{1}\right|>1\right\}$ is a component of the Fatou set of $f$, in all senses, because all $f^{k}$ are holomorphic there and converge uniformly on compacts to a constant map to $r=[0: 1: 0]$.

Levi flat cone $L:=\left\{\left|z_{0}\right|=\left|z_{1}\right|\right\}$ is a Julia set of $f$. It contains one of two indeterminacy points of $f$, namely $q=[0: 0: 1]$. A connected component $\Omega$ of $\mathbb{P}^{2} \backslash L$ different from $\Phi$ carries a more interesting information about $f^{k}$. First of all remark that as a mapping from $U_{2}$ to $U_{2}$ our iterate writes as

$$
\begin{aligned}
f^{k}:\left(w_{1}, w_{2}\right) & \rightarrow\left(\frac{w_{2}^{2^{k}-1}}{w_{1}^{2^{k}-2}}, \frac{w_{2}^{2^{k+1}-1}}{w_{1}^{2^{k+1}-2}}\right) \rightarrow(0,0)=q \\
\text { on } \quad\left\{\left|w_{2}\right|<\left|w_{1}\right|\right\} & =\left\{\left|u_{1}\right|<1, u_{2} \neq 0\right\} .
\end{aligned}
$$

Therefore the second component $\Phi_{s}^{2}$ of the strong Fatou set contains the domain $\Omega \backslash\left\{u_{2}=0\right\}$. Since it is easy from (8.5) to see that $f^{k}$ on compacts in $\Omega \backslash\left\{u_{2}=0\right\}$ converge to $q$, and on the punctured disk $\Delta^{*}:=\left\{u_{2}=0,0<\left|u_{1}\right|<1\right\}$ to $p$, we conclude that $\Phi_{s}^{2}=\Phi_{w}^{2}=\Omega \backslash\left\{u_{2}=0\right\}$. Remark that the second component $\Phi^{2}$ of $f$ in the sense of [8] is smaller, namely it is equal to $\Omega \backslash\left(\left\{u_{2}=0\right\} \cup\left\{u_{1}=0\right\}\right)$, because the projective line $l_{1}:=\left\{z_{1}=0\right\}$ is the preimage of $I_{f}$ (and of all $I_{f^{k}}$ ) under $f$. Now let us turn to the second component $\Phi_{\Gamma}^{2}$ of the $\Gamma$-Fatou set of $f$.

Lemma 8.4. For a fixed $0<\varepsilon<1$ the volumes of graphs of $f_{k}$ over the bidisk $\Delta_{\varepsilon}^{2} \subset U_{0}$ centered at $p$ are uniformly bounded. In particular $\Phi_{\Gamma}^{2}=\Omega$.

Proof. To estimate the volume of $\Gamma_{f_{k}}$ over a neighborhood of $p$ we use coordinates $u_{1}, u_{2}$ and representation (8.5). In these coordinates $\Delta_{\varepsilon}^{2}=\{u:\|u\|<\varepsilon\}$. Since $f^{k}$ preserves the vertical lines $\left\{u_{1}=\right.$ const $\}$ we can simplify our computations assuming that $f$ takes values in $\Delta \times \mathbb{P}^{1}$, the last being equipped with the Hermitian 
metric form $\omega=\omega_{1}+\omega_{2}=\frac{i}{2} d z_{1} \wedge d \bar{z}_{1}+\frac{i}{2} \frac{d z_{2} \wedge d \bar{z}_{2}}{\left(1+\left|z_{2}\right|^{2}\right)^{2}}$. Now we get

$$
\begin{aligned}
\left(f^{k}\right)^{*} \omega= & \frac{i}{2}\left[2^{2 k}\left|u_{1}\right|^{2^{k+1}-2}+\frac{\left(1-2^{k}\right)^{2}\left|u_{1}\right|^{2^{k+1}}-4}{\left(\left|u_{1}\right|^{2^{k+1}-2}+\left|u_{2}\right|^{2}\right)^{2}}\right] d u_{1} \wedge d \bar{u}_{1} \\
& +\frac{i}{2}\left|u_{1}\right|^{2^{k+1}-2} \frac{d u_{2} \wedge d \bar{u}_{2}}{\left(\left|u_{1}\right|^{2^{k+1}-2}+\left|u_{2}\right|^{2}\right)^{2}} \\
& +\frac{i}{2}\left(1-2^{k}\right) \bar{u}_{1}^{-2^{k}}\left|u_{1}\right|^{2^{k+2}-4} \frac{d u_{2} \wedge d \bar{u}_{1}}{\left(\left|u_{1}\right|^{2^{k+1}-2}+\left|u_{2}\right|^{2}\right)^{2}} \\
& +\frac{i}{2}\left(1-2^{k}\right) u_{1}^{-2^{k}}\left|u_{1}\right|^{2^{k+2}-4} \frac{d u_{1} \wedge d \bar{u}_{2}}{\left(\left|u_{1}\right|^{2^{k+1}-2}+\left|u_{2}\right|^{2}\right)^{2}}
\end{aligned}
$$

Therefore

$$
\begin{aligned}
& \int_{\Delta_{\varepsilon}^{2}}\left(f^{k}\right)^{*} \omega \wedge d d^{c}\|u\|^{2} \\
& =4 \pi^{2} \int_{0}^{\varepsilon} \int_{0}^{\varepsilon}\left[2^{2 k} r_{1}^{2^{k+1}-1}+\left(1-2^{k}\right)^{2} \frac{r_{1}^{2^{k+1}-3}}{\left(r_{1}^{2^{k+1}-2}+r_{2}^{2}\right)^{2}}+\frac{r_{1}^{2^{k+1}-1}}{\left(r_{1}^{2^{k+1}-2}+r_{2}^{2}\right)^{2}}\right] d r_{1} r_{2} d r_{2} \\
& \leq \frac{4 \pi^{2} \varepsilon^{2^{k+1}}}{2^{2^{k+1}}-2 k}+2 \pi^{2} 2^{2 k} \int_{0}^{\varepsilon} \int_{0}^{\varepsilon^{2}} \frac{r_{1}^{2^{k+1}-3} t}{\left(r_{1}^{2^{k+1}-2}+t\right)^{2}} d t d r_{1}+2 \pi^{2} 2^{2 k} \int_{0}^{\varepsilon} \int_{0}^{\varepsilon^{2}} \frac{r_{1}^{2^{k+1}-1}}{\left(r_{1}^{2^{k+1}-2}+t\right)^{2}} d t d r_{1} \\
& \leq 2 \pi^{2} 2^{2 k} \int_{0}^{\varepsilon} \int_{0}^{\varepsilon^{2}} \frac{r_{1}^{2^{k+1}-3}}{r_{1}^{2^{k+1}-2}+t} d t d r_{1}+2 \pi^{2} \int_{0}^{\varepsilon}\left(\left.\frac{-1}{r_{1}^{2^{k+1}-2}+t}\right|_{0} ^{\varepsilon^{2}} r_{1}^{2^{k+1}-1}\right) d r_{1} \\
& \leq \pi^{2} 2^{3 k+1} \int_{0}^{\varepsilon} r_{1}^{2^{k+1}-3} \ln \frac{1}{r_{1}} d r_{1}+2 \pi^{2} \int_{0}^{\varepsilon} r_{1} d r_{1} .
\end{aligned}
$$

The second term is bounded and does not tend to zero as $k \rightarrow+\infty$. The first for $0<\varepsilon<1$ can be obviously bounded by

$$
\pi^{2} 2^{3 k+1} \int_{0}^{\varepsilon} r^{2^{k+1}-4} d r=\frac{\pi^{2} 2^{3 k+1}}{2^{k+1}-3} \varepsilon^{2^{k+1}-3} \rightarrow 0
$$


as $k \rightarrow+\infty$. And finally

$$
\begin{aligned}
\int_{\Delta_{\varepsilon}^{2}}\left(f^{k}\right)^{*} \omega= & \int_{\Delta_{\varepsilon}^{2}} \frac{2^{2 k}\left|u_{1}\right|^{2^{k+2}-4}}{\left(\left|u_{1}\right|^{2^{k+1}-2}+\left|u_{2}\right|^{2}\right)^{2}}+\frac{\left(2^{k}-1\right)^{2}\left|u_{1}\right|^{2^{k+2}-6}\left|u_{2}\right|^{2}}{\left(\left|u_{1}\right|^{2^{k+1}-2}+\left|u_{2}\right|^{2}\right)^{4}} \\
& +\frac{2\left(2^{k}-1\right)^{2}\left|u_{1}\right|^{2^{k+3}-8}\left|u_{2}\right|^{2}}{\left|u_{1}\right|^{2^{k+1}}\left(\left|u_{1}\right|^{2^{k+1}-2}+\left|u_{2}\right|^{2}\right)^{4}} d^{4} m(u) \\
\leq & \int_{\Delta_{\varepsilon}^{2}} \frac{2^{2 k+2}\left|u_{1}\right|^{2^{k+2}-6}}{\left(\left|u_{1}\right|^{2^{k+1}-2}+\left|u_{2}\right|^{2}\right)^{2}} d^{4} m(u) \\
\approx & 2^{2 k+2} \int_{0}^{\varepsilon} \int_{0}^{\varepsilon^{2}} \frac{r_{1}^{2^{k+2}-5}}{\left(r_{1}^{2^{k+1}-2}+t\right)^{2}} d t d r_{1} \\
\leq & 2^{2 k+2} \int_{0}^{\varepsilon} r^{2^{k+1}-3} d r=\frac{2^{2 k+2}}{2^{k+1}-2} \varepsilon^{2^{k+1}-2} \rightarrow 0
\end{aligned}
$$

as $k \rightarrow+\infty$. Therefore the lemma is proved.

Remark that the integral of $\left(f^{k}\right)^{*} \omega^{2}$ degenerates, as it should be, because $f^{k}$ $\Gamma$-converge on $\Omega$ to a constant map. And to the contrary the integral of $\left(f^{k}\right)^{*} \omega$ does not degenerate, moreover has order $\varepsilon^{2}$. That means that bubbling takes place over all points of the disk $\Delta^{*}=\left\{u_{2}=0,0<\left|u_{1}\right|<1\right\}$. We see that for our map one has

$$
\Phi \subset \Phi_{w}=\Phi_{s} \subset \Phi_{\Gamma}
$$

and inclusions are strict.

Finally let us see what is going on over the indeterminacy point $p=(0,0)$ in coordinates $\left(u_{1}, u_{2}\right)$. Blowing up $2^{k}-1$ times at zero we see that $f^{k}$ stays degenerate on all exceptional curves except for the last one, which it send onto $l_{1}=\left\{u_{1}=0\right\}$. In appropriate coordinates $v_{1}, v_{2}$ on the last blow up $f^{k}$ writes as

$$
\left\{\begin{array}{l}
u_{1}=v_{1}^{2^{k}} \\
u_{2}=v_{2} .
\end{array}\right.
$$

Therefore the dynamical picture over $p$ can be described as follows. Let $\hat{\Omega}$ be an infinite blow up of $\Omega$ over $p$ and let $C=\bigcup_{i=1}^{\infty} C_{i}$ be the Nori string of rational curves on $\hat{\Omega}$ over $p$. Then every $f^{k}$ lifts to a holomorphic map $\hat{f}^{k}: \hat{\Omega} \rightarrow \mathbb{P}^{2}$ which is constantly equal to $q$ on every $C_{i}$ except $C_{2^{k}-1}$. The last curve sends bijectively onto the line $l_{1}$. At the same time in the sense of divisors (currents) $f^{k}\left(C_{2^{k}-1}\right)=2^{k} l_{1}$. 
Remark 8.5. a) One can in the same way produce mappings of any given degree with the same properties as in Example 8.3. It is sufficient to take

$$
f:\left[z_{0}: z_{1}: z_{2}\right] \rightarrow\left[z_{0}^{d} z_{1}: z_{1}^{d+1}: z_{0}^{d} z_{2}\right]
$$

b) Let us quote the result of Maegava, see [30], which shows that under an additional assumption of "algebraic stability" of the dominant rational self-map $f$ the Fatou set of [8] coincides with $\Phi_{s}$ and $\Phi_{w}$.

\section{References}

[1] C. Araujo, Rationally connected varieties, Contemp. Math. 388 (2006), 1-16.

[2] D. BARLET, Espace analytique reduit des cycles analytiques complexes compacts d'un espace analytique complexe de dimension finie, In: "Fonct. de plusieurs variables complexes", Seminar Norguet IX, Lect. Notes Math., Vol. 482, 1975, 1-157.

[3] W. Barth, C. Peters and A. Van de Ven, "Compact Complex Surfaces", Springer Verlag, 1984.

[4] E. BEDFORD and B. A. TAYLOR, A new capacity for plurisubharmonic functions, Acta Math. 149 (1982), 1-40.

[5] E. BISHOP, Conditions for the analyticity of certain sets, Michigan Math. J. 11 (1964), 289-304

[6] B. CARTAN and P. Thullen, Zur Theorie der Singularitäten der Funktionen mehrerer Verandenlichen: Regularitäts und Konvergenzbereiche, Math. Ann. 106 (1932), 617-647.

[7] F. DoCQUiER and H. GRAUerT, Levisches Problem und Rungescher Satz für Teilgebiete Steinscher Mannigfaltikeiten, Math. Ann. 140 (1960), 94-123.

[8] J.-E. ForNAESS and N. SibONy, Complex dynamics in higher dimension. II, In: "Modern Methods in Complex Analysis (Princeton, NJ, 1992)", T. Bloom et al. (eds.), Ann. of Math. Stud., Vol. 137, Princeton Univ. Press, Princeton, NJ, 1995, 135-182.

[9] A. FuJIKI, Closedness of the Douady space of compact Kähler spaces, Publ. Res. Inst. Math. Sci., Kyoto Univ. 14 (1978), 1-52.

[10] H. Fujimoto, On families of meromorfphic maps into the complex projective space, Nagoya Math. J. 54 (1974), 21-51.

[11] P. GAUdUCHON, Les metriques standard d'une surface a premier nombre de Betti pair, Astérisque 126 (1985), 129-135.

[12] P. GRIFFITHS and J. KING, Nevanlinna theory and holomorphic mappings between algebraic varieties, Acta Math. 130 (1973), 145-220.

[13] M. GREEN, The hyperbolicity of the complement of $2 n+1$ hyperplanes in general position in $\mathbb{P}^{n}$, and related results, Proc. Amer. Math. Soc. 66 (1977), 109-113.

[14] R. Harvey, Holomorphic chains and their boundaries, Proc. Symp. Pure. Math. 30, part 1 (1977), 309-382.

[15] R. HARVEY and B. Shiffman, A characterization of holomorphic chains, Ann. of Math. 99 (1974), 553-587.

[16] L. HÖRMANDER, "An Introduction to Complex Analysis in Several Variables", Third edition, North-Holland Mathematical Library, Vol. 7, North-Holland Publishing Co., Amsterdam, 1990.

[17] S. IVASHKOVICH, The Hartogs-type extension theorem for the meromorphic maps into compact Kähler manifolds, Invent. Math. 109 (1992), 47-54. 
[18] S. IVASHKovich, On convergence properties of meromorphic function and mappings (Russian), In: "Complex Analysis in Modern Mathematics", B. Shabat memorial volume, FAZIS, Moscow, 2001, 133-151, see also arXiv:math/9804009.

[19] S. IVASHKOVICH, Extension properties of meromorphic mappings with values in nonKahler complex manifolds, Ann. of Math. 160 (2004), 795-837.

[20] S. IVASHKOVICH, On fixed points of rational self-maps of mplex projective plane, Indiana Univ. Math. J. 60 (2011), 803-812.

[21] S. IVASHKovich and V. SHEVChishin, Structure of the moduli space in a neighborhood of a cusp-curve and meromorphic hulls, Sbornik Math. 189 (1998), 1335-1359.

[22] S. IVASHKOVICH and V. SHEVCHISHIN, Deformations of non-compact complex curves and envelopes of meromorphy of spheres, Invent. Math. 136 (1999), 571-602.

[23] S. Ivashrovich and V. Shevchishin, Gromov compactness theorem for J-complex curves with boundary, Int. Math. Res. Not. 22 (2000), 1167-1206.

[24] G. Julia, Sur les familles de fonctions analytiques de plusieurs variables, Acta Math. 47 (1925), 53-115.

[25] J. KING, A residue formula for complex subvarieties, Proc. Carolina Conf. on Holomorphic Mappings and Minimal Surfaces (Chapel Hill, N.C., 1970) Dept. of Math., Univ. of North Carolina, 43-56.

[26] P. Kiernan, Hyperbolically imbedded spaces and the big Picard theorem, Math. Ann. 204 (1973), 203-209.

[27] C. Kiselman, Sur la définition de l'opérateur de Monge-Ampère complexe, In: "Analyse complexe, Proc. Journ. Fermat - Journ SMF", Toulouse 1983, Lecture Notes in Math., Springer, Berlin, Vol. 1094, 1984, 139-150.

[28] S. KOBAYASHI, Intrinsic distances, measures and geometric function theory, Bull. Amer. Math. Soc. 82 (1976), 357-416.

[29] E. LEVI, Studi sui punti singolari essenziali delle funzioni analitiche di due o più variabili complesse, Ann. Mat. Pura Appl. 17 (1910), 61-87.

[30] K. MAEGAWA, Ascoli-Arzela type theorem for rational iterations of the complex projective plane, Proc. Amer. Math. Soc. 136 (2008), 2875-2879.

[31] C. Meneghini, Renormalizing iterated repelling germs of $\mathbb{C}^{2}$, Mat. Vesnik 56 (2004), $85-90$.

[32] F. NEJI, Equicontinuous families of meromorphic mappings with values in compact complex surfaces, Complex Var. Elliptic Equ. 57 (2012), 1339-1347.

[33] T. Nishino, Sur les familles de surfaces analytiques, J. Math. Kyoto Univ. 1 (1962), 357377.

[34] J. Noguchi and J. Winkelman, Holomorphic curves and integral points of divisors, Math. Z. 239 (2002), 593-610.

[35] T. RANSFord, "Potential Theory in the Complex Plane", London Mathematical Society Student Texts 28, Cambridge Univ. Press, Cambridge, UK, 1995.

[36] O. RIEMENSCHNEIDER, Über den Flächeninhalt analytischer Mengen und die Erzeugung kpseudokonvexer Gebiete, Invent. Math. 2 (1967), 307-331.

[37] H. RUTISHAUSER, Über Folgen und Scharen von analytischen und meromorphen Funktionen mehrerer Variabeln, sowie von analytischen Abbildungen, Acta Math. 83 (1950), 249-325.

[38] W. SAXER, Über die normalen Scharen meromorpher Funktionen mehrerer Variablen, Comm. Math. Helv. 4 (1932), 256-267.

[39] Y.-T. SIU, Extension of meromorphic maps into Kähler manifolds, Ann. of Math. 102 (1975), 421-462.

[40] Y.-T. SiU, Every Stein subvariety admits a Stein neighborhood, Invent. Math. 38 (1976), 89-100.

[41] H. SKodA, Sous-ensembles analytiques d'ordre fini ou infini dans $\mathbb{C}^{n}$, Bull. Soc. Math. France 100 (1972), 353-408.

[42] W. STOLL, Normal families of non-negative divisors, Math. Z. 84 (1964), 154-218. 
[43] G. Stolzenberg, "Volumes, Limits and Extension of Analytic Varieties", Springer Verlag, 1966.

[44] M. Zaidenberg, Picard's theorem and hyperbolicity, Siberian. Math. J. 24 (1983), 858867.

Université de Lille-1

UFR de Mathématiques

59655 Villeneuve d'Ascq, France

and

IAPMM of Ukrainian NAS

Naukova, $3 / \mathrm{b}$

290053 Lviv, Ukraine

ivachkov@math.univ-lille1.fr

Université de Gabès

ISSI de Gabès

rue Slaheddine El Ayoubi

6032 Gabès, Tunisie

nejimath@gmail.com 